\title{
Progress and perspective on advanced cloaking metasurfaces: from invisibility to intelligent antennas
}

\author{
Stefano Vellucci ${ }^{1, *}$, Alessio Monti $^{2}$, Mirko Barbuto $^{2}$, Alessandro Toscano $^{1}$, and Filiberto Bilotti ${ }^{1}$ \\ ${ }^{1}$ Department of Engineering, ROMA TRE University, Via Vito Volterra 62, Rome 00146, Italy \\ 2 Engineering Department, "Niccolò Cusano" University, Via Don Carlo Gnocchi 3, Rome 00166, Italy
}

Received: 23 November 2020 / Accepted: 20 December 2020

\begin{abstract}
Among the different cloaking applications proposed in the literature, the antenna framework has emerged as one of the most fruitful and mature field. In particular, mantle cloaking approach has proven to be a powerful tool for enabling unprecedented possibilities in antenna design. Here, we provide a review of the most significant works in the field of electromagnetic invisibility for antenna applications, demonstrating the versatility of cloaking metasurfaces in antenna scenarios. We also discuss our recent results and investigations on the design of advanced cloaking metasurfaces equipped with electronic components and circuits and able enriching the antenna intelligence.
\end{abstract}

Keywords: Metasurfaces / cloaking / antennas / intelligent / cognitive / self-adaptive

\section{Introduction}

Arguably, electromagnetic invisibility is one of the most catching applications enabled by metamaterials [1-9]. Although the problem of electromagnetic invisibility has been investigated by the scientific community before the metamaterial era, for instance in the fields of non-radiating sources [10,11], invisible particles [12,13], or low-scattering antennas [14-16], in the last decades the research has spread significantly with the development of several different invisibility approaches. In general, an invisibility device, also referred to as a "cloak", aims making an object completely undetectable to external sensors within a specific frequency range. Ideally, a cloaked object is characterized by a massive suppression of its scattering for all the observation angles, all the polarizations and within a broad frequency range; however, in real scenarios, these constraints can be relaxed. In fact, none of the passive metamaterial-based approaches developed so far in the literature enables achieving a perfect cloaking condition within a non-zero bandwidth [17]. However, each of them comes with specific advantages and constraints, they can be properly selected depending on the application of interest.

For instance, transformation-based cloaking [18-22] exploits the principles of transformation electrodynamics [23-26] to bend and stretch the space surrounding the

\footnotetext{
* e-mail: stefano.vellucci@uniroma3.it
}

object to hide and re-route the power flow around the concealed volume. The propagating wave is forced to follow curved coordinates when interacting with a coating metamaterial-based shell, making the inner region effectively invisible to external excitation. Since the coated object does not interact with the electromagnetic energy, it does not scatter, that is, it is truly invisible.

Although very elegant, the transformation-based cloaking approach comes with some theoretical constraints and inherent fabrication challenges. First, the metamaterial shell must me realized by a medium characterized by strong inhomogeneous and anisotropic distributions of permeability and permittivity, which made the design and the fabrication process very difficult, and inherently sensitive to small fabrication tolerances [27]. Second, the requirement for extreme constitutive parameters is usually associated with strong material losses, as a consequence of Kramers-Kronig relations [28], which implies absorption and forward scattering for the optical theorem. Finally, if not relying on Non-Euclidian transformation electrodynamics [29], a transformation-based cloak requires superluminal propagation for the rays that go through the shell to travel, in equal time, larger distance compared to the ray in vacuum, drastically limiting the operational bandwidth of the device [30,31]. For these reasons, the experimental realizations of transformation-based cloaks usually simplify the design introducing some specific assumptions, such as one polarization of interest, the introduction of mismatch at the boundary between the vacuum and the shell, or a relaxed phase match constraints [32]. 
More robust results can be achieved by slightly modifying the cloaking strategy and introducing quasiconformal mapping technique in transformation optics [33]. Several experimental realizations exploiting this carpet cloaking technique have been proved [34,35], although, arguably, the concealed object is made appears flat rather than truly invisible.

A different approach is introduced by the transmission-line cloaking technique $[36,37]$, which aims at guiding the incident field through a transmission line network designed to be impedance-matched to free space. Since the energy is guided through a network that does not necessary wrap the concealed object [38-40], superluminal propagation is not required and the cloak can be made extremely broadband [41]. The main disadvantage of this approach is that the concealed object must be complementary to the network, that is, should fit the transmission line fishnet $[42,43]$ (with some exceptions as in Ref. [41]); as a consequence, the cloak is inherently bulky and massive, although the manufacturing is usually quite straightforward.

It is worth noticing that cloaking approaches discuss so far are impractical for sensing and antennas applications, since the concealed object is electromagnetically isolated from the external environment due to the re-direction of the energy all around. The coated object is, thus, unable to receive or transmit electromagnetic energy, and its functionality is inevitably compromised. In these scenarios, however, a different method can be applied.

Plasmonic [44] and mantle cloaking [45] techniques are, in fact, based on a radically different paradigm exploiting the scattering cancellation principle. Here, the concealed object is coated by either a bulk or a thin shell aiming at canceling out just the dominant scattering terms in the multipole expansion of the field originally scattered by the object to hide. Since invisibility is achieved when the induced dipole moments of the cloak and the object are equal in magnitude but out-of-phase, the scattering cancellation principle allows to not isolate the concealed volume from the surrounding environment. Instead, scattering from the object is required to have a proper destructive interference effect leading to the cloaking functionality. This approach is, thus, particularly suited for sensing and antenna applications [46-48].

However, differently from previously mentioned cloaking techniques, scattering cancellation cloaks are designed to hide specific objects, and cannot work for others objects with different shapes or material properties. Although this is not necessarily a disadvantage, it represents a major difference that should be considered during the cloak design process. More relevant is the limitation related to the electrical size of the object. In fact, the scattering cancellation technique is not well suited for very large object compared to the operative wavelength since the number of scattering modes that come into play in the description of the scattering response of the object rapidly increases with the size of the object itself [49-53]. Although multi-layered cloaks aiming at minimizing the contribution of each scattering mode for each layer can be exploited [54-57], for electrically large objects the complexity of the design of the cloak drastically increases, as well the complexity of the analytical models due to the mutualcoupling between the metasurface layers [55].

Compared to other cloaking techniques, the design of scattering cancellation cloaks is rater straightforward, and they can be made by either using coat made of bulk plasmonic metamaterials or ultrathin conformal metasurfaces. In plasmonic cloaking, the cloak is made out of isotropic and homogeneous materials [58-64], and it is particularly suited for applications at optical frequencies due to the broad availability of plasmonic materials [65-67]. At microwave frequencies, instead, the mantle cloaking approach tends is preferred since the cloak can be implemented through ultrathin and conformal metasurfaces made of simple patterned metallic surfaces realizable using conventional PCB fabrication techniques [68-73].

Compared to plasmonic cloaking, instead of employing a metamaterial shell in which an opposite dipole moment is induced, here, the ultrathin metasurface is designed such that the scattering field produced by the currents induced onto the surface cancels out with the field scattered by the object to hide. The effect of the surface is to introduce a discontinuity to the tangential component of the magnetic field across the interface, which can be controlled by the value of the surface impedance $Z_{s}(\Omega / \mathrm{sq})$ of the metasurface, defined as the ratio between the tangential component of the electric field and the surface current density, that is, $\boldsymbol{E}_{t a n}=Z_{s} \boldsymbol{J}[45,68,74,75]$. In general, $Z_{s}$ is a complex quantity described by a real part, due to the losses of the materials composing the metasurface, and an imaginary part describing the energy stored [76]. Since at microwave frequencies mantle cloaking metasurfaces can be safely assumed lossless, due to the high conductivity of the patterned metallic sheet, the $Z_{s}$ can be described by just the value of its surface reactance $X_{s}\left(\Omega / \mathrm{s}_{\mathrm{q}}\right)$. The advantages of a cloak that is low weight, ultra-thin, conformal, easily manufacturable, and which does not compromise the electromagnetic functionality of the concealed object, makes the mantle cloaking approach a rather versatile technique for antenna applications. For the reader convenience, in Figure 1a comparison between the main cloaking approaches in terms of performances and design characteristics is reported.

In the following, we present a survey of previous results and ongoing investigations in the field of mantle cloaking metasurfaces for antenna applications. Specifically, we focus on the recent trends foreseeing the use of advanced cloaking metasurfaces that allow increasing the degrees of freedom available to the antenna designer and making the cloaked radiating systems more intelligent. After a brief review of the basic concepts and theoretical aspects of the mantle cloaking approach (Sect. 2), we discuss the main and established applications of passive, linear, and time-invariant mantle cloaks for antennas (Sect. 3). Then, we move to the recent possibilities enabled by cloaking metasurfaces integrating electronic circuits (Sect. 4) and, finally, discuss the challenges and perspectives of this new antenna design paradigm (Sect. 5). 


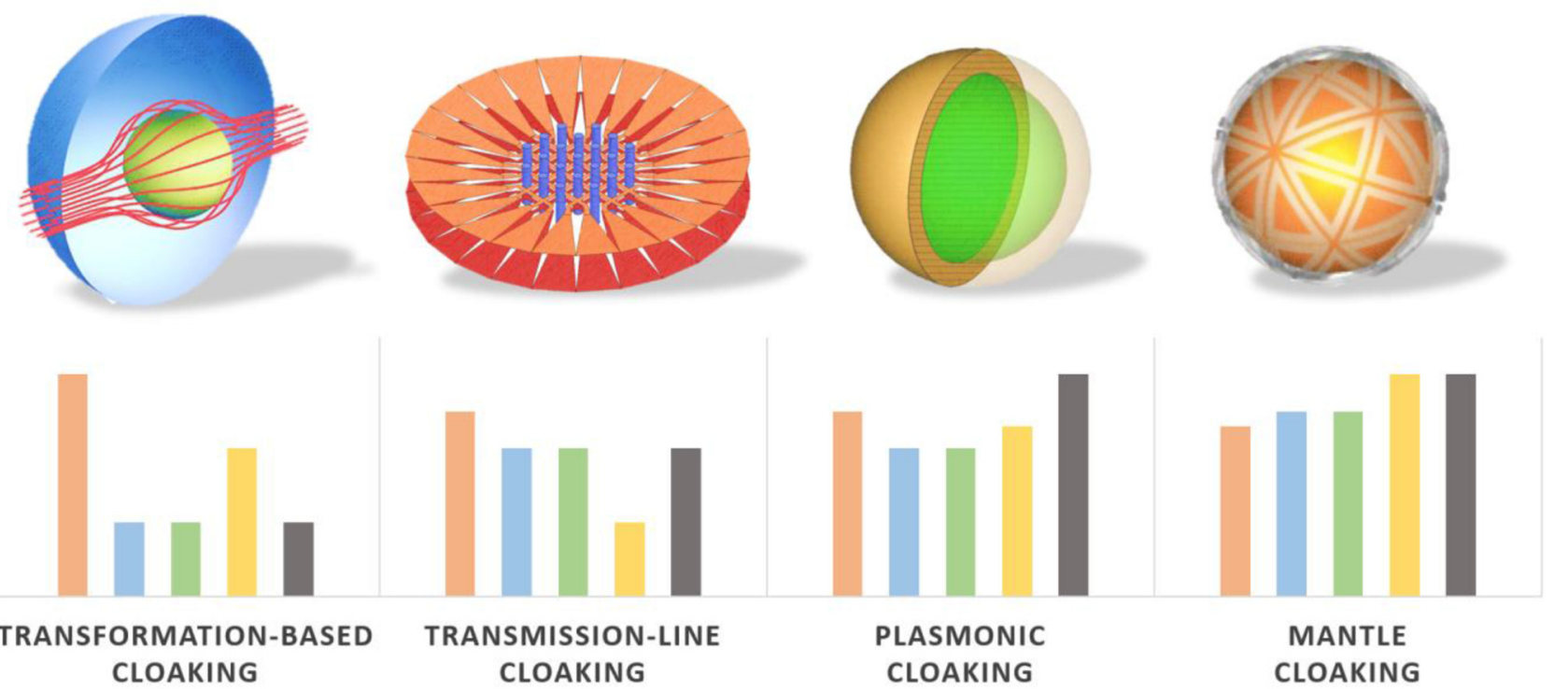

\section{Scattering reduction $\square$ Bandwidth $\square$ Feasibility Low-profile $\square$ Isolation}

Fig. 1. Qualitative comparison between the main cloaking approaches proposed in the literature in terms of scattering reduction performance (red column), theoretical bandwidth (blue column), feasibility and ease of implementation (green column), low-profile (yellow column), and electromagnetic isolation of the concealed object (black column).

\section{Principle of electromagnetic invisibility for antennas trough metasurfaces}

The mantle cloaking technique has been introduced by Prof. A. Alù in [45] for the case of spherical objects and quickly extended to $1 \mathrm{D}$ and $2 \mathrm{D}$ problems involving dielectric planar slabs, and infinitely extended cylinders [68]. The latter scenario is particularly relevant in the antenna field since, in first approximation, applies to the case of wired antennas such as dipoles, monopoles, strips, etc. Thus, here, we briefly summarize the main concepts behind the mantle cloaking approach for the case of an infinite circular metallic cylinder, offering a physical insight into the working principle.

The problem of scattering reduction from an elongated cylindrical object through ultrathin coating layers can be rigorously formulated starting from the Mie theory and deriving closed-form equations in the quasi-static regime. More recently, the mantle cloaking approach has been reformulated by considering a matching problem involving radial transmission lines $[77,78]$. Though the latter approach offers a more straightforward solution to the cloaking problem, especially when higher order modes are considered, here we analyze the classical formulation based on the generalization of the Mie theory to a metallic cylinder coated by a surface impedance sheet.

Let us consider, under the $e^{\mathrm{j} \omega \mathrm{t}}$ time convention, a perfectly conducting infinite wire of radius $a$ illuminated by a monochromatic $T M_{z}$ plane wave (i.e., with the electric field parallel to the cylinder axis $z$ ). The $T M_{z}$ polarization is considered here since it is the most relevant ones in the case of wired antenna oriented along the $z$-axis. The wire is also coated by a conformal ideal metasurface of radius $a_{c}$, placed onto a dielectric support of permittivity $\varepsilon_{c}$, such that $\gamma=a_{c} / a$. Let us also assume that the metasurface is ultrathin, and realized by an array of particles without any natural magnetic properties. In the limit of zero thickness, thus, the metasurface can support only electric surface current and its surface impedance $Z_{s}$ can be described by an homogenized scalar quantity [76].

From the Mie theory, the incident and scattered fields can be represented by expansion in cylindrical harmonics as [79]:

See equation below

$$
\boldsymbol{E}_{z}^{t o t}=\left\{\begin{array}{cl}
\hat{\boldsymbol{z}} E_{0} \sum_{n=-\infty}^{\infty} j^{-n}\left[a_{n}^{T M} J_{n}\left(k_{c} \rho\right)+b_{n}^{T M} Y_{n}\left(k_{c} \rho\right)\right] e^{j n \varphi}, & a<\rho<a_{c} \\
\hat{\boldsymbol{z}} E_{0} \sum_{n=-\infty}^{\infty} j^{-n}\left[J_{n}\left(k_{0} \rho\right)+c_{n}^{T M} H_{n}^{(1)}\left(k_{0} \rho\right)\right] e^{j n \varphi}, & \rho>a_{c}
\end{array}\right\}
$$


where the components of the magnetic field can be derived as $\boldsymbol{H}=-\frac{1}{j \omega \mu} \nabla \times \boldsymbol{E}$, and, $J_{n}(\cdot)$ and $H_{n}^{(1)}(\cdot)$ are the cylindrical Bessel and Hankel functions of the first kind and order $n$, while $k_{0}$ and $k_{c}$ are the wavenumbers in free space and inside the coating dielectric support, respectively. Thus, the scattered field is conveniently expressed as a discrete sum of cylindrical harmonics with complex amplitudes $c_{n}^{T M}$. Compared to the expressions in $[49,68]$, the amplitude coefficients of the scattered $T E$ cylindrical waves $c_{n}^{T E}$ are missing since we have still introduced the assumption of $T M$ plane wave excitation and, thus, the scattering coefficients $c_{n}^{T E}$ are identically null.

In general, the total scattering cross-section can be evaluated as the superposition of normal modes, each weighted by the appropriate coefficients $c_{n}^{T M}$ (and $c_{n}^{T E}$, eventually). However, for the case of an infinitely long wire (i.e., a wire very long compared to its diameter), it is more convenient to normalize the scattering cross section to the unit of length, that is, in terms of scattering width (SW) as [49]:

$$
\sigma=\frac{4}{k_{0}} \sum_{n=-\infty}^{\infty}\left|c_{n}^{T M}\right|^{2} .
$$

Interestingly, the scattering width is a sum of infinite terms of yet unknown coefficients $c_{n}^{T M}$. For the case of a thin wire (i.e., with diameter small compared to the operative wavelength), the scattering coefficients above the fundamental order are negligible [49] and the scattered field is dominated by the zeroth-order mode $c_{0}^{T M}$, which is sufficient to represent the induced current. The thin wire assumption (or, in other terms, the electrically small condition) is safely assumed since the wired antennas considered in the following are always characterized by a reduced cross-section needed to maintain their resonant behavior.

The expression of the unknown amplitude coefficient $c_{0}^{T M}$ can be found by enforcing the continuity of the tangential components of the electric and magnetic fields at $\rho=a$, and the boundary condition of an electricallypolarizable metasurface at $\rho=a_{c}$ [68], which reads as: $\left.\boldsymbol{E}_{t a n}\right|_{\rho=a_{c}^{ \pm}}=Z_{s} \hat{\boldsymbol{r}} \times\left(\left.\boldsymbol{H}_{t a n}\right|_{\rho=a_{c}^{+}}-\left.\boldsymbol{H}_{t a n}\right|_{\rho=a_{c}^{-}}\right)$. The expression of the $c_{0}^{T M}$ coefficient is thus dependent on the value of $Z_{s}$. By vanishing this quantity, it is possible deriving an exact formula returning the value of the cloaking surface impedance $Z_{s}$. By expanding the Bessel and the Hankel functions with proper Taylor series, this expression can be easily simplified to:

$$
Z_{s}=-j \frac{4 a_{c} \mu_{0} \omega \ln \left(\frac{a}{a_{c}}\right)}{2\left(a_{c} k_{0}\right)^{2}\left(\varepsilon_{c}-1\right) \ln \left(\frac{a}{a_{c}}\right)+\varepsilon_{c}\left(a_{c} k_{0}\right)^{2}+4} .
$$

Interestingly, a similar closed-form expression is also obtained to achieve a camouflaging or illusion effect, that is, to make the coated wire appears as if made of a different material or characterized by different geometrical parameters [80]. In that case, $Z_{s}$ is chosen to return the desired non-zero $c_{0}^{T M}$ of the targeted object.

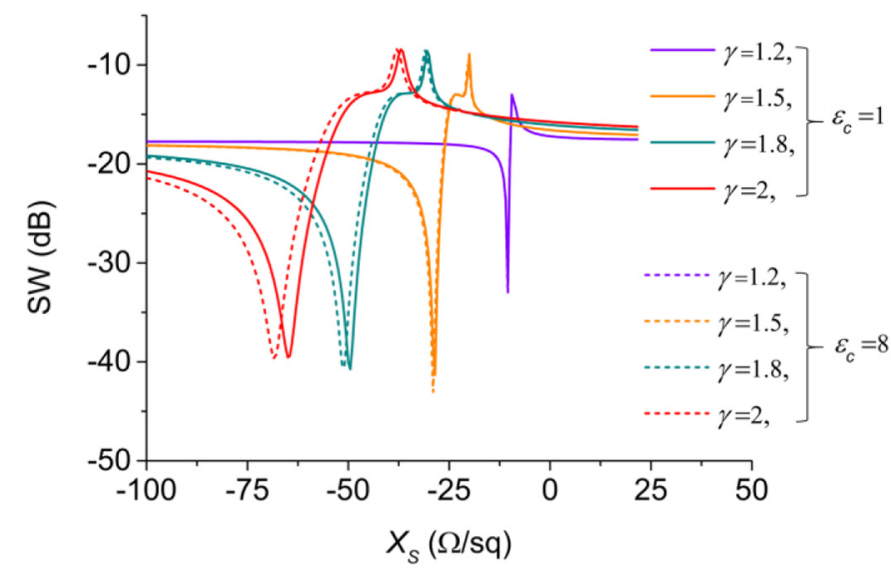

Fig. 2. Theoretical SW of a $2 \mathrm{D}$ metallic wire as the surface impedance of the coating metasurface changes, for different combination of the cloak radius and of the substrate permittivity. The radius of the wire is $a=\lambda_{0} / 20$.

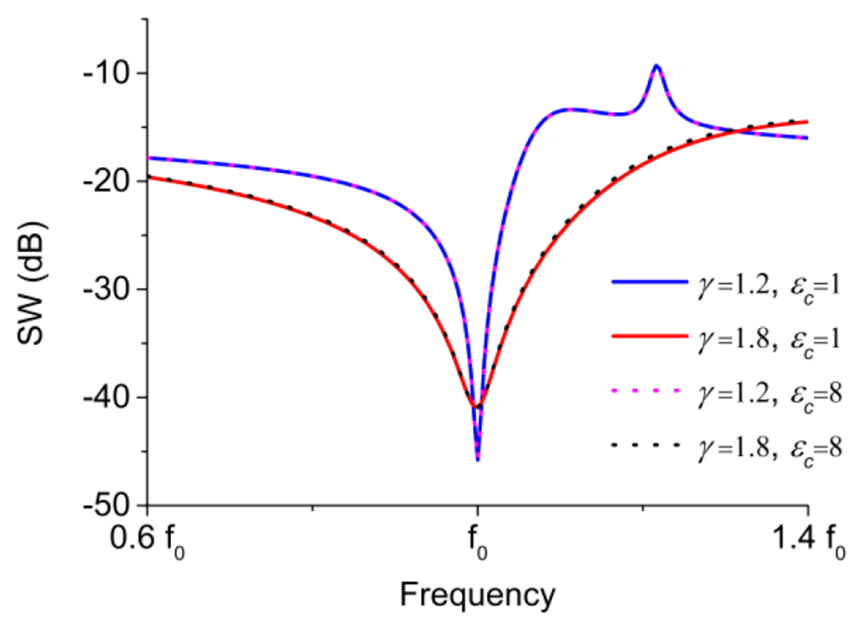

Fig. 3. Analytical results for the frequency variation of the SW of a $2 \mathrm{D}$ metallic wire for different values of the cloak radius and permittivity. The radius of the wire is $a=\lambda_{0} / 20$, while $Z_{s}=-j$ $10.34 / 10.36 / 49.59 / 51.20$ for $\left[\gamma=1.2, \varepsilon_{c}=1\right], \quad\left[\gamma=1.2, \varepsilon_{c}=8\right]$, $\left[\gamma=1.8, \varepsilon_{c}=1\right],\left[\gamma=1.8, \varepsilon_{c}=8\right]$, respectively.

The availability of a closed-form formula for the cloaking condition allows making some considerations on the design of cloaks for wire devices. As can be appreciated from Figure 2, for the case of a metallic wire of radius $a=\lambda_{0} / 20$, the $Z_{s}$ cloaking value depends on both the dimension and electromagnetic properties of the coating supporting medium. Increasing either the cloak radius or the medium permittivity shifts the $Z_{s}$ towards higher absolute values, although less in the latter case. In this way, it is possible to tune the value of the cloaking surface impedance towards realistic values, easily implementable at microwave frequencies, and compatible with the conformal design of the cover.

More importantly, from Figure 3, we can see that increasing the cloak radius significantly increases the 


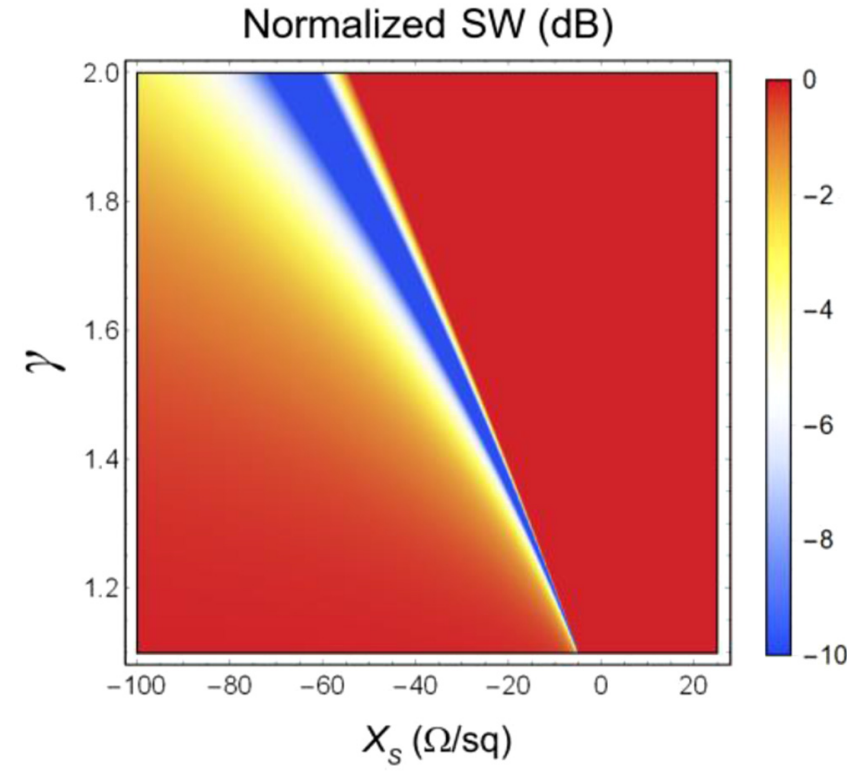

Fig. 4. Theoretical SW of a coated $2 \mathrm{D}$ metallic wire normalized to the SW in the uncoated case, as $\gamma=a_{c} / a$ and the surface impedance of the coating metasurface change. The radius of the wire is $a=\lambda_{0} / 20$, while the permittivity of the substrate $\varepsilon_{c}$, is equal to one.

cloaking bandwidth, at the expense of a reduction of the SW at the design frequency $f_{0}$. This is a major point in antenna applications. In antenna scenarios, it is usually required to reduce the total scattering within specific bandwidth. The use of cloaks with large radii, thus, can improve the frequency performance of the cloak, leading to the desired bandwidth coverage [81]. Moreover, it is worth mentioning that, since the mantle cloaking technique exploits a non-resonant cloaking approach, it can provide larger cloaking bandwidth compared to other cloaking approaches [17].

Finally, in Figure 4 the SW of a coated metallic wire of radius $a=\lambda_{0} / 20$ evaluated at the design frequency $f_{0}$ and normalized to the wire SW in the uncoated case when varying both $\gamma$ and the coat surface impedance $Z_{s}$ is reported. We can see that increasing the cloak radius allows broadening the range of surface impedance values reducing the wire SW (more than $10 \mathrm{~dB}$ ). This confirms the relevant conclusion that increasing the cloak radius lets relax the $Z_{s}$ cloaking requirement, introducing a smoother variation in the cloaking surface impedance value and, thus, a more robust sensitivity over fabrication tolerances.

We notice that non-dispersive surface impedance and permittivity have been considered in the analytical formulation. As can be appreciated from Figure 3, the value of the permittivity seems not affecting the cloaking bandwidth (although it affects the $Z_{s}$ cloaking value, as reported in Fig. 2). However, in a realistic scenario, the inherent dispersion of both the surface impedance and the permittivity do affects the frequency performance of the cloak. In particular, the use of a dielectric substrate characterized by a large value of the permittivity can increase the inherent dispersion of the metasurface [82], reducing the cloaking bandwidth. Furthermore, losses from the dielectric substrate would further deteriorate the performance of the cloak [83] and, thus, low loss materials should be chosen for realistic cloak design.

In essence, the analytical design procedure discussed allows synthesizing the desired value of the cloaking surface impedance once fixed the radius and the material properties of the supporting medium around the wire. In the design process, different degrees of freedom can be exploited to suit the specific requirements of the application of interest. However, the reported analytical results represent a first approximation of the cloaking problem in the case of wired antennas. In fact, two main and fundamental differences apply. First, wire antennas are finite-length structure with usually a length comparable with the incident wavelength. The Mie theory, thus, does not strictly applies and the analytical formulas return just preliminary design values. Nevertheless, in the literature, it has been repeatedly proved that still there is a very good agreement between the analytical and numerical/measured results, even for the case of finite wire/road with a length of one/two-wavelength [64,70]. Secondly, wire antennas are closed on a resistive load placed at their input port. The value of the load strongly affect the scattering response of the device and, thus, the surface impedance required to cloak the antenna/sensor [84]. A numerical optimization procedure is, thus, required to check for the optimum value of the cloaking surface impedance [81]. Albeit some limitations of the analytical design procedure exist in antenna applications, in the following, we will illustrate several representative examples of cloaking antenna systems, proving the validity of the mantle cloaking approach for antenna scenarios. The physics mechanism behind the mantle cloaking/scattering cancellation approach remains, as remarked by the fact that it can be exploited to cloak also non-canonical structures [60].

\section{Antenna applications of cloaking metasurfaces}

Among the different cloaking applications proposed in the literature, antenna scenarios have been one of the most investigated frames. The opportunity of making antennas or structural elements of a radiating system invisible, or modifying the scattering property of an antenna device have always attracted great attention, as demonstrated by the very first applications conceived when metamaterialbased cloaking approaches have been first introduced $[46,47,85]$. Due to its inherent advantages in terms of feasibility and flexibility of the cloak design, especially when dealing with elongated structures, the potentialities of mantle cloaking in antennas have been deeply explored. Here, we proposed a survey on the main antenna applications conceived in the last decade, starting with the ones dealing with cloaking of structural or passive objects placed nearby of an antenna. Then, we move to the studies about the effect of a cloak on the scattering and absorption characteristics of receiving antenna systems, and, finally, we discuss the applications exploiting cloaking 
systems for the reduction of the blockage effects and mutual coupling between antennas placed in close proximity.

\subsection{Cloaking of passive objects}

As well known, in an antenna system, the presence of structural or passive elements close to an antenna (up to a few wavelengths) can perturb the near-field distribution of the radiated electromagnetic field and, thus, both the electrical and radiative properties of the antenna. The possibility of making such elements invisible to the antenna radiation is, thus, a very appealing scenario.

Arguably, this idea has been conceived before the cloaking era, through the introduction of the so-called hard- and soft-surfaces [16], although with some limitations. In such a technique, to reduce the forward scattering from an elongated thin object, for example, metallic cylinders, struts, or masts, the device cross-section is modified to give it an oblong shape, and it is then coated by a longitudinally corrugated dielectric material to obtain a hard boundary condition $[86,87]$. The main limitations of this approach are the requirement of a modification of the original shape of the coated object, and its strong dependence on the angle of arrival of the impinging electromagnetic wave. However, as demonstrated in [88], this approach can be successfully used to significantly mitigate the deterioration effects on the sidelobe, directivity, and cross-polarization performance of a center-fed parabolic reflector, by replacing the conventional metallic struts with low scattering ones. In particular, the experimental results show significant improvement on the sidelobe level of a Ku-band center-fed reflector antenna supported by four rhombus-shaped metallic struts covered by corrugated dielectric layers, compared to the uncoated struts case, thanks to a more than $6 \mathrm{~dB}$ radar cross-section (RCS) reduction of the struts when covered by the design hard-surface.

As remarked, the hard- and soft-surface approach suffers from inherent drawbacks that limit the possible antenna applications. Instead, the mantle cloaking approach is characterized by great flexibility when dealing with cylindrical metallic objects, with the possibility of applying a coating cloak even on preexisting structures. For instance, in [89], mantle cloaking devices for enhancing the performance of the transmitting antenna mounted on a nanosatellite platform has been investigated. The interference effects appearing on the communication system of small-satellites is a well-known issue, mostly related to the extremely small dimension of the device and the consequent placement of the payloads in the near field of the antenna [90,91]. The case of a CubeSat for weather measurement equipped with Langmuir probes mounted on deployable metallic cylindrical booms was considered (Fig. 5a). The antenna for communication with the radio base station, or other satellites of the constellation in the small-satellite network, exploits a cross-dipole antenna operating in the UHF spectrum. As can be appreciated from Figures 6a and 6b, due to the small dimension of the nanosatellite, the metallic booms equipped with the sensors

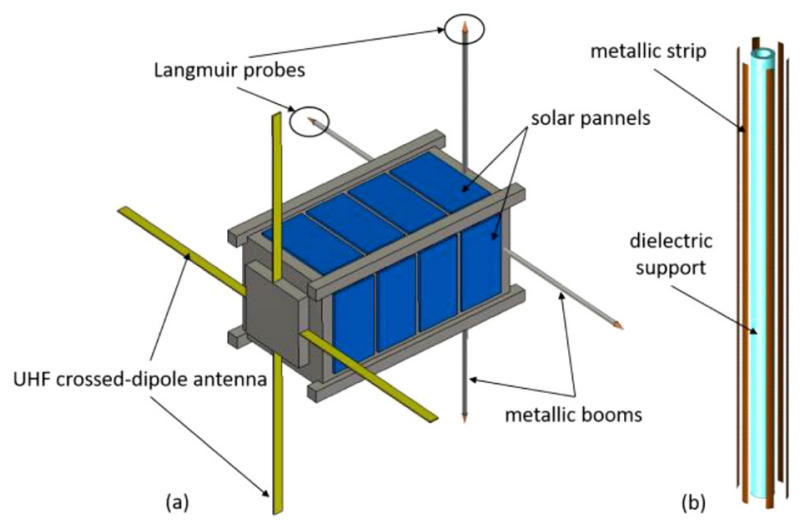

Fig. 5. (a) Sketch of the CubeSat system for space wheatear measurements. (b) Details of the mantle cloak applied to the metallic booms (adapted from [89]).

drastically affect the radiative performance of the antenna once deployed, compared to the almost isotropic radiation pattern of the antenna system in the undeployed scenario (Fig. 6a). When deployed (Fig. 6b), the metallic supports introduce strong nulls, sharpening the radiation pattern. Consequentially, the communication link becomes sensitive to the spatial orientation of the satellite, which is not equipped with an alignment control system, as usually happens in CubeSats to reduce the onboard power consumption. Equipping the metallic booms with a properly designed mantle cloaking device allows to massively mitigate the deteriorating effects, as visible from (Fig. 6c), restoring the original radiation pattern. Here, the metallic supports are wounded around a dielectric support, specifically designed for space application (e.g. with low outgassing and water absorption), where longitudinal metallic strips synthesizing an inductive metasurface are printed on (Fig. 5b). As can be appreciated, the cloak is made of a rather straightforward design that neither modifies the supporting structure nor perturbs the operation of the sensors.

The strategy utilized for hiding the deployable booms of the satellite, in principle, can also be used to hide other payloads, enabling the possibility to further reduce the overall dimension of the nano-satellite [92], or conceiving antenna systems with reduced power consumption, thanks to the increased efficiency of the antennas. Other interesting applications include the opportunity of designing multi-band antenna systems operating at different frequencies (e.g. UHF and VHF) equipped on the same satellite platform, that are made invisible one to the other [93].

Another interesting example showing the potentialities of mantle cloaks for hiding passive objects obstructing radiating elements is reported in [72]. In this case, the scenario of an array of metallic roads placed in front of a pyramidal horn antenna at a distance of the order of some fraction of the operative wavelength has been analyzed, as illustrated in Figure 7. Clearly, the presence of the roads in the near-field region of the antenna dramatically affects the 


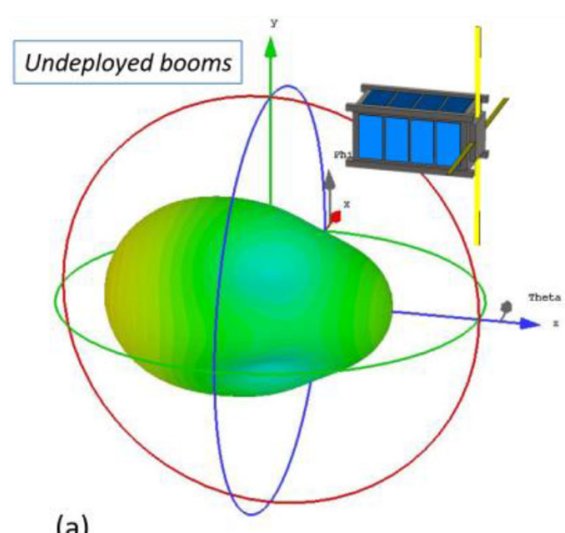

(a)

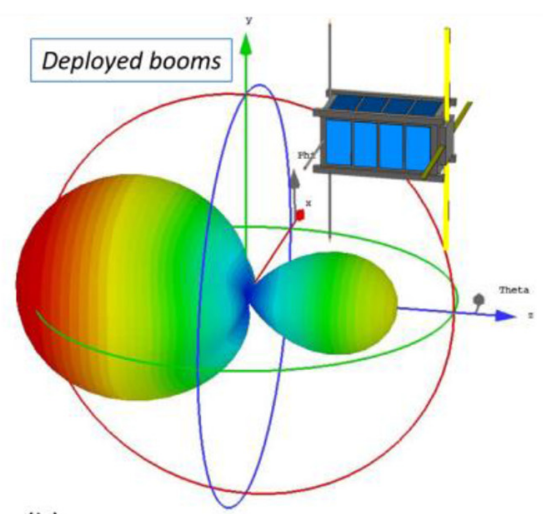

(b)

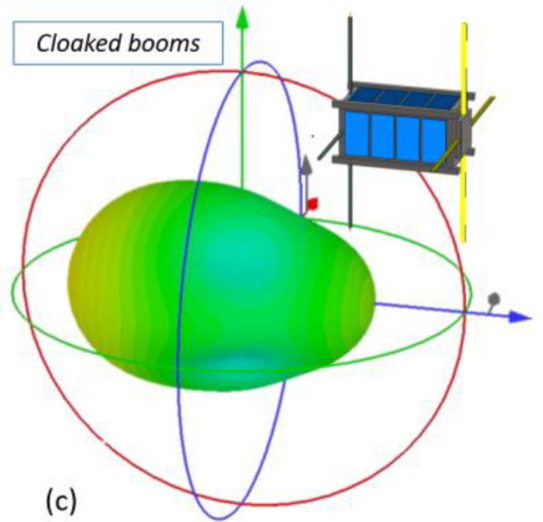

(c)

Fig. 6. Radiation patterns of the of the CubeSat system in the case of (a) undeployed sensors, (b) deployed sensors, and (c) deployed sensors once the metallic booms are coated by the mantle cloak (adapted from [89]).

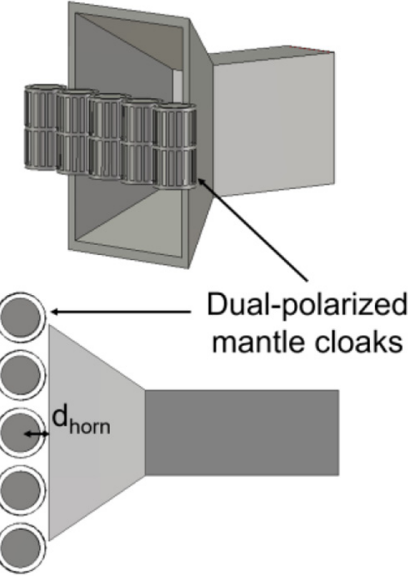

Fig. 7. Sketch of a horn antenna obstructed by metallic cylinders placed in front of the aperture made invisible trough coating mantle cloaks. Prospective and top views (adapted from [72]).

electrical and radiative performances of the antenna. In particular, the waves scattered by the roads introduce high reflections, worsening the antenna impedance matching, as can be appreciated from the amplitude of the reflection coefficient reported in Figure 8a (continuous blue line), compared to the performance achieved without the array of roads (dotted black curve). To restore the original antenna performance, a dual-polarized mantle cloak made of rectangular unit-cells has been designed, like the one reported in Figure 7 (refer to [72] for more details on the mantle cloak design). A dual-polarized metasurface has been used to design a cloaking system independent from the spatial orientation of the roads, with respect to the horn at the central frequency of operation antenna aperture orientation. Thanks to the use of a rectangular unit-cell, the mantle cloak synthesizes two different values for the surface impedance, which have been engineered to return the two cloaking values needed to cloak the roads for both the $T M$ and $T E$ polarization. As can be seen from Figure 8

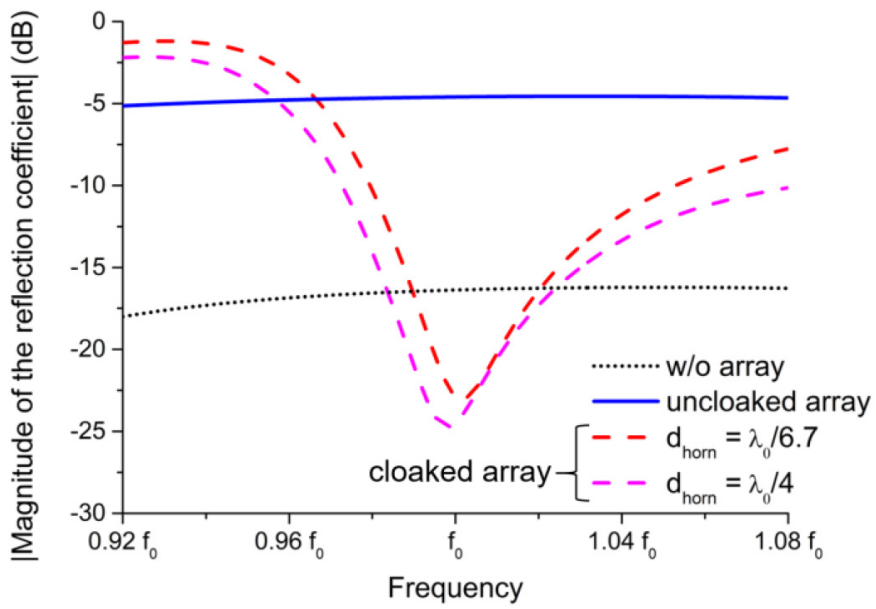

Fig. 8. Magnitude of the horn antenna reflection coefficient in the absence and presence of bare and cloaked array of cylinders for different values of the distance between the antenna aperture and cylinders $\left(d_{\text {horn }}\right)$ (adapted from $\left.[72]\right)$.

(dashed lines), once placed around the roads, the mantle cloaks can restore the electrical characteristics of the horn, almost independently from the distance between the cylinders and the antenna aperture. At the same time, the gain performances are also dramatically improved if compared to the behavior in the uncloaked scenario [72]. It is worth mentioning that, actually, a similar scenario has been also considered in [42], exploiting a transmission linecloak as a cloaking device. However, in this case, the cloak was rather bulky and more complicated compared to the discussed solution, and worked just for one single polarization.

Overall, the discussed results confirm the practical and straightforward implementation of mantle cloaking devices able to hide passive objects placed in proximity of antennas, enabling unpreceded solutions for the improvement of the antennas performance obstructed by metallic objects, or for the enhancement of the performance of antennas on small satellites. 


\subsection{Cloaking of receiving antennas}

As previously mentioned, one of the main advantages of mantle cloaking over other invisibility techniques is related to the possibility of coating an object without isolating it from the surrounding environment. Thanks to this peculiar characteristic, the design of low scattering sensors have been proposed [46], but also the possibility of modifying the scattering and absorption characteristics of an antenna has been deeply explored. For instance, in [84], it was shown how, by exploiting properly designed mantle cloaks, it is possible to minimize the scattering from receiving antennas or sensors maximizing, at the same time, their absorption efficiency.

From the theory of receiving antennas, when an antenna load is conjugate matched with its input impedance maximum absorption and resonance are achieved [94]. This condition implies that the total absorbed and scattered cross-section (or, in other terms, the absorbed and scattered power [94]) are equal, that is, $\sigma_{t}^{A}=\sigma_{t}^{S}$ [95]. A resonating antenna, thus, has poor performance as a non-invasive sensor/probe, since an ideal sensor should scatter a reduced amount of power to limit the perturbation of the field it is sensing. In the case of a short dipole antenna, a minimum scattering condition can be nevertheless achieved by introducing the so-called antiresonance condition [96], that is, when $X_{L}=-4 X_{i n}$ for fixed $R_{L}$, where $X_{L}$ and $X_{i n}$ are the load and input reactance of the antenna, respectively. At the minimum scattering condition, thus, minimum perturbation of the surrounding field is achieved, although at the expense of a reduction of the absorbed power level, which compromises the efficiency of the antenna as a receiving radiator [96]. In particular, for small dipole antennas, fundamental limitations on the maximum scattering for a given level of absorption exist [84,95], and these bounds can be reached just for a specific combination of $R_{L}$ and $X_{L}$. However, in [84], it has been shown that by exploiting a scattering cancellation-based cloak it is possible to ensure that, when $X_{L}=-X_{i n}$, for every value of $R_{L}$, almost all the physical bound of maximum absorption efficiency for a given absorption level is reached (where the absorption efficiency reads as $\left.\eta_{e f f}=\sigma_{t}^{A} / \sigma_{t}^{S}\right)$. This means that just adjusting the antenna load with a potentiometer the behavior of the antenna can be controlled, tuning it from a high-scattering mode, for an optimal conjugate matched receiver (maximum absorption), to a low scattering sensor.

Although the theoretical analysis is strictly valid just for short dipoles, the same concept can be still applied to half-wavelength dipole antennas to increase their absorption efficiency at resonance [84]. In Figure 9, the absorption efficiency $\left(\eta_{\text {eff }}\right)$ vs total absorption $\left(\sigma_{t}^{A}\right)$ for the cases of a bare and coated half-wavelength dipole antenna is reported. Here, the shaded region of the plot is forbidden by the limitations discussed before, while any point inside the white area is theoretically allowed. As can be appreciated, by sweeping the load resistance $\left(R_{L}\right)$ for both configurations, much larger absorption efficiency is obtained in the coted scenario (red continuous curve). For the case of the coated dipole, the absorption efficiency lies almost on the optimal bound, whereas for bare antenna

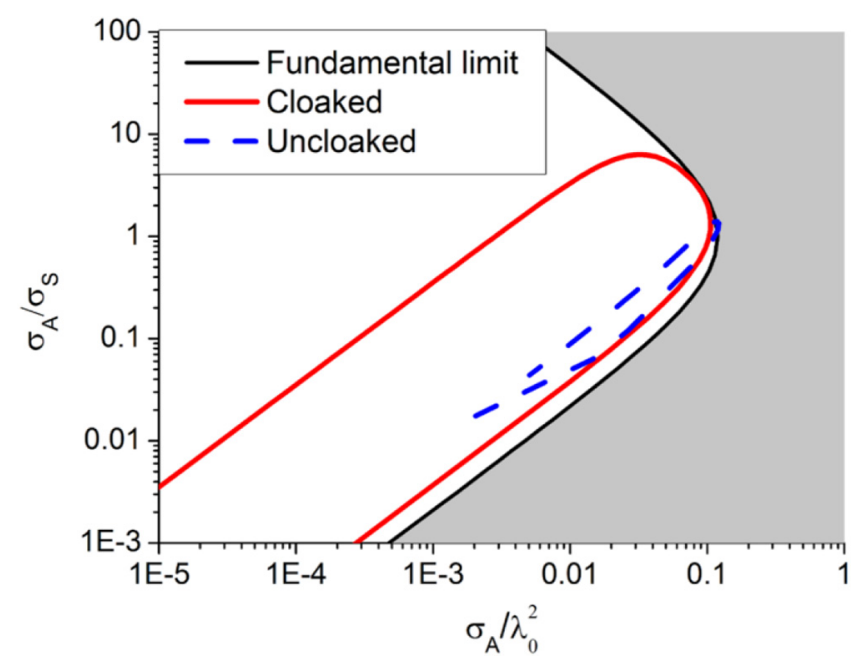

Fig. 9. Absorption efficiency $\left(\eta_{\text {eff }}=\sigma_{A} / \sigma_{S}\right)$ vs total absorption $\left(\sigma_{A}\right)$ for the cases of a bare and coated half-wavelength dipole antenna when sweeping the load resistance $\left(R_{L}\right)$.

the absorption efficiency is always below the unitary value. This remarkable increase in absorption efficiency is accomplished using a simple and feasible mantle cloak made of an inductive metasurface on a dielectric substrate (refer to [84] for more details), optimized minimizing the structural scattering from the antenna, that is, cloaking the antenna in the open-circuit condition. Thus, it is evident that by canceling the dipole structural scattering, the range where maximum absorption and maximum absorption efficiency are achieved at resonance is significantly broaden. This means that by coating a resonating halfwavelength dipole antenna with a properly designed mantle cloak and loading it with a variable resistive load, it is possible to ensure the maximum possible absorption level for any level of efficiency, enabling a simple way to vary the antenna absorption efficiency while guaranteeing optimal operation, as confirmed by the results reported in Figure 10.

The discussed results represent a significant advancement towards the realization of highly efficient, simple, and tunable sensing antennas, and open up interesting possibilities for the design of antenna devices with tunable and controllable absorption and scattering properties.

\subsection{Cloaking for blockage and mutual-coupling reduction}

One of the most useful applications of mantle cloaking for antennas lies in the possibility to mutually hide transmitting antennas placed at small electrical distances. Although theoretically introduced in [85] using ideal transformationoptic cloaks, this concept reached the technological maturity only with the advent of the mantle cloaks, that allows designing conformal, realistic and high-performance cloaking devices.

In particular, in [97], for the first time, the design of two conformal and simple metasurfaces printed on a dielectric 


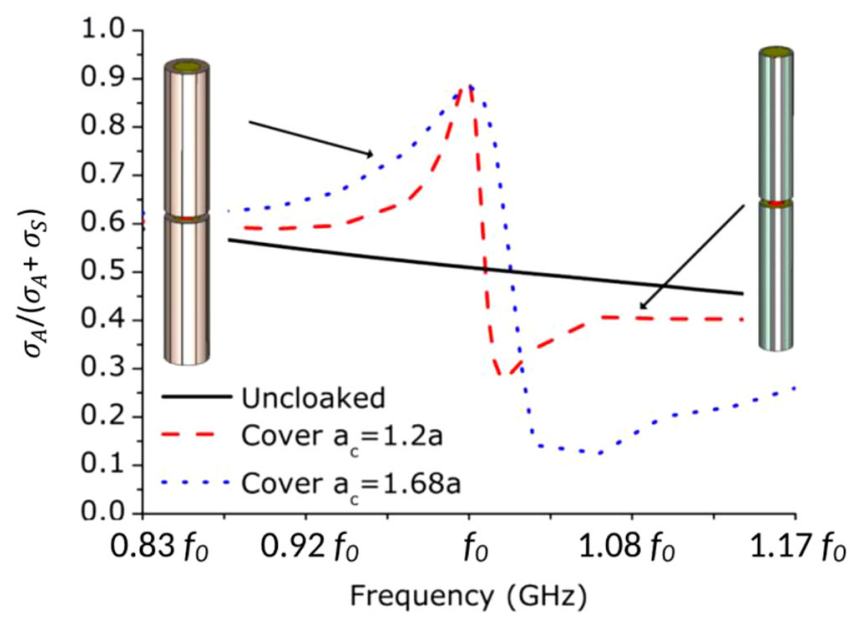

Fig. 10. $\sigma_{A} /\left(\sigma_{S}+\sigma_{A}\right)$ vs frequency for different cloak design coating a half-wavelength dipole antenna. Curves are obtained for a fixed value of the load resistance $\left(R_{L}\right)$.

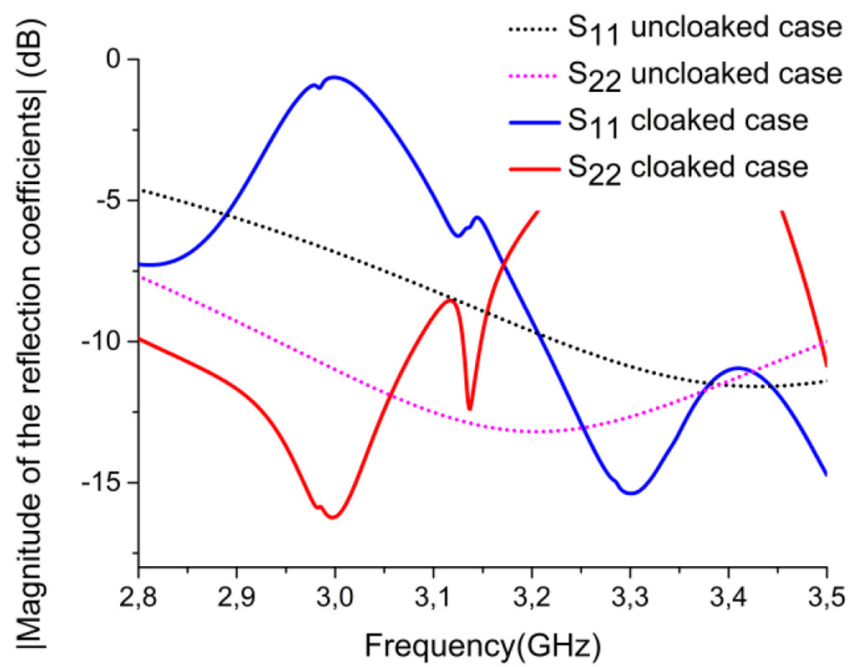

Fig. 11. Magnitude of the reflection coefficients at the input port of the first and second antenna in the uncloaked case (dotted lines) and of the first and second antenna in the cloaked case (continuous lines) (adapted form [97]).

substrate has been proposed to significantly reduce the mutual blockage effect between two transmitting halfwavelength dipole antennas, placed in extremely close electrical proximity. Two dipoles operating at $f_{1}=$ $3.33 \mathrm{GHz}$ and $f_{2}=3.07 \mathrm{GHz}$, respectively, were considered. As reported in Figure 11, when the two antennas are placed at close proximity, in particular at just $d=\lambda / 10$ at $3 \mathrm{GHz}$, their reflection coefficients performances are severely compromised and lose their classical resonant behaviour. However, introducing the mantle cloaks allows largely mitigating the deteriorating effect, restoring the resonant behaviour around the resonant frequency of the antennas (i.e., around 3.07 and $3.33 \mathrm{GHz}$ ). In this scenario, the mantle cloaks have been designed to mutually hide the antennas, that is, the first antenna is covered with a mantle cloak operating at $f_{1}$, while the second one is cloaked at $f_{2}$. The beneficial effect of the cloaks is also confirmed by the 2D gain plots reported in Figure 12, where the gain patterns on the $\mathrm{H}$ - and E-planes for the isolated, uncloaked, and cloaked scenario are illustrated. As can be appreciated, when the mantle cloaks are applied the antennas operate as if they were isolated.

This design principle has been experimentally proved, for multiple antennas tightly packed together in a highly dense telecommunication platform. As a proof of concept, in [81], two monopole antennas operating for $3 \mathrm{G}$ and $4 \mathrm{G}$ mobile communications were placed nearby (nearly touching). Thanks to the presence of a mantle cloaking device around the antenna operating for $4 \mathrm{G}$ communication, the two antenna systems were shown to operate as if they were isolated, paving the way to new co-siting strategies for multiple antenna systems handling different services and installed in overcrowded platforms, such as communication towers and ship trees. In this case, due to the significantly different working frequencies (UMTS services in the band 1.9.-2.2 GHz, and LTE low band 790$860 \mathrm{MHz}$ ) and, thus, the different length of the monopoles, just the longer LTE monopole represents a strong scatterer for the UMTS monopole, perturbing its electrical and radiative performance. Conversely, the shorter UMTS antenna introduces a marginal degradation effect on the LTE monopole. Therefore, it was required to design just one invisibility device able to conceal the LTE monopole at the operating frequency of the UMTS antenna. This was achieved by exploiting a simple cloak design made of a hollow dielectric cylinder of FR4, coated by a capacitive metasurface consisting of three horizontal metallic strips wounding the dielectric support. Remarkably, thanks to the large dimension of the cloak radius $\left(a_{c}=2 \times a\right)$, the cloaking device was able to hide the LTE monopole within the whole bandwidth of operation of the UMTS services, as reported in the measured results shown in Figure 13. Moreover, the mantle cloak was shown to not perturbing the performance of the coated LTE antenna, making this a backward-compatible solution for pre-existing antenna systems.

A different mantle cloak design for a similar application has been proposed instead in [98]. Here, both the two monopole antennas required a cloaking device to efficiently operate, and a more complicated composition for the mantle cloaks comprising arrays of subwavelength electric and magnetic resonators was used. To suppress both the mutual coupling and mutual blockage effects caused by the close space between the antennas, a multi-layered cloaking metasurface was designed. By tailoring the dispersive properties of the metasurface layers, the authors showed that both the electrical and radiative characteristics of the antennas were restored to the isolated case.

The same approach was used to design also a multiband mantle cloak capable of cloaking a monopole antenna at multiple frequencies [99]. To show that, a monopole antenna operating at $f_{0}=2.4 \mathrm{GHz}$ was coated by the multiband mantle cloak working at $f_{1}=3.5 \mathrm{GHz}$ and $f_{2}=$ $5.2 \mathrm{GHz}$. For testing the effectiveness of the cloak, an uncoated dual-band monopole antenna was placed in the proximity of the first radiator, showing that comparable 

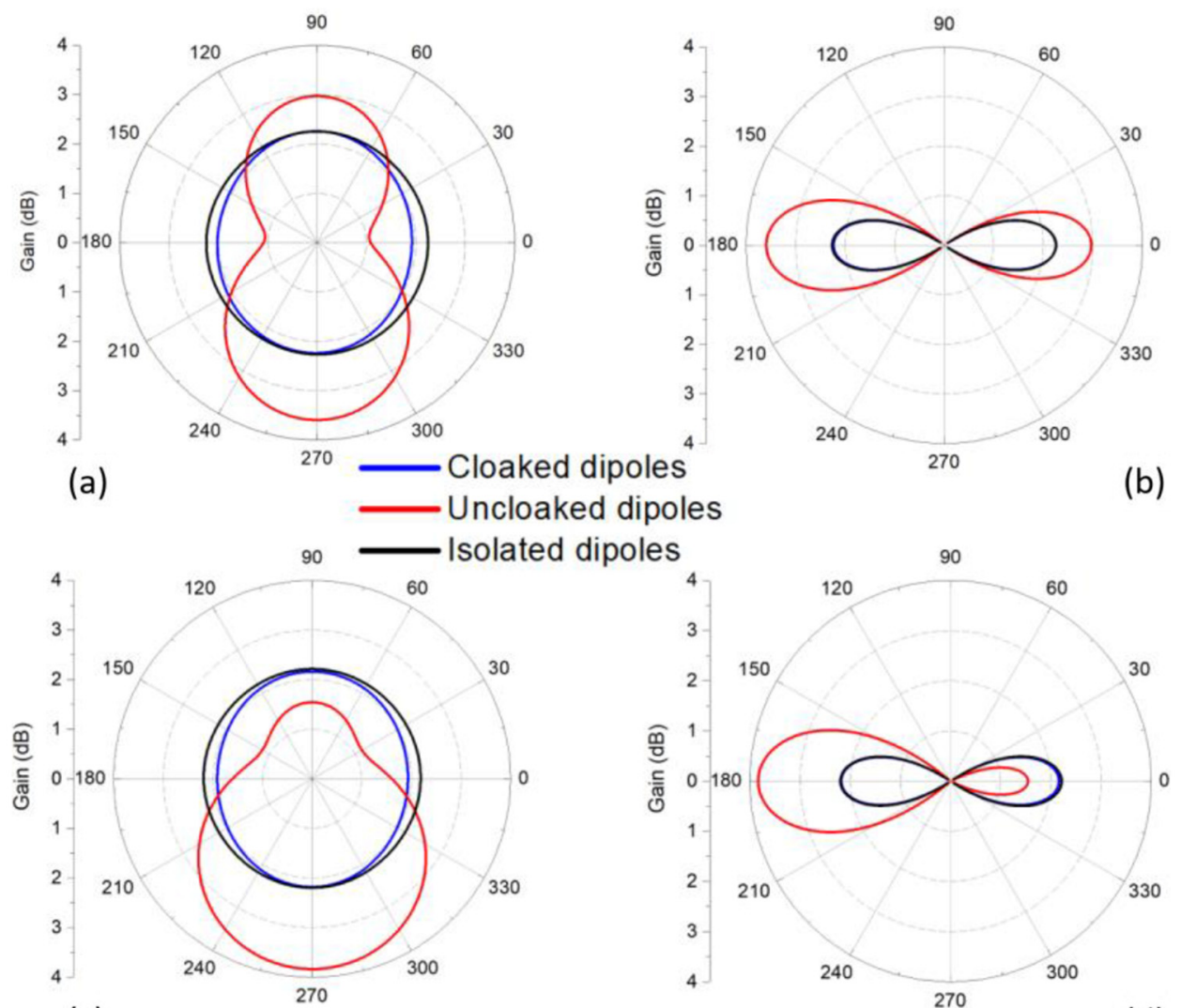

(b)

(c)

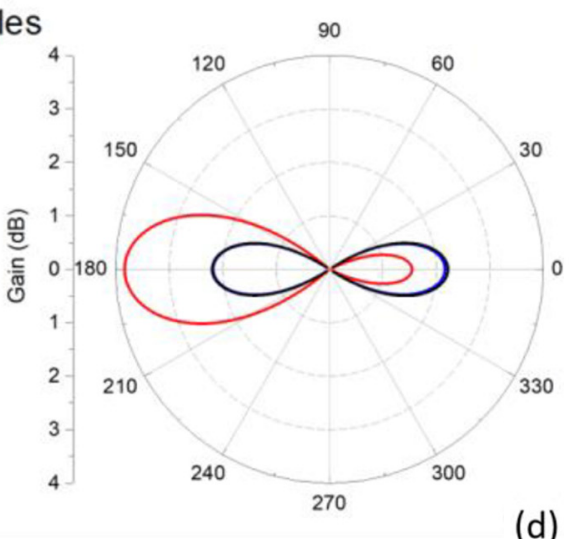

Fig. 12. 2D gain plots of the first antenna at $3.298 \mathrm{GHz}$ on the (a) H-plane and (b) E-plane in the case of cloaked dipoles (blue lines), the uncloaked scenario (red lines), and the isolated one (black lines). (c) and (d) show the same quantities for the second antenna at $2.970 \mathrm{GHz}$ (adapted form [97]).

behaviour for the S-parameter and radiation patterns in the cloaked and absence of the first antenna were achieved.

In [100], the concept of antenna blockage reduction using mantle cloaking devices was further expanded, through the realization of a dual-polarized mantle cloak for a dipole antenna. A cloaking metasurface made of patch unit-cell was designed to hide a dipole antenna placed in front of a pyramidal horn. Similar to what numerically reported in [72] for the case of blockage from passive objects, here, the mantle cloak was shown to hide the dipole antenna at the working frequency of the horn for both TM and TE polarization.

The possibility of designing compact multi-band radiating structures has not been limited to the cases of monopoles and dipoles but also extended to the case of nonsymmetric radiating devices such as strip antennas. In [101], elliptical metasurfaces made of conformal vertical or horizontal metallic strips have been introduced to reduce the mutual coupling between two closely spaced strip dipoles. However, compared to the previous solutions, here the length of the strips was modified compared to the uncoated scenario to consider the resonance frequency shifts due to the presence of the cloak. Importantly, in [102] the use of elliptical metasurface cloaks to restore the intrinsic properties of radiating systems was extended to the case of printed antennas with strip inclusions. As a proof of concept, two microstrip-fed monopoles working at slightly different frequencies and partially embedded in the same substrate were considered. Elliptical mantle cloaks were numerically demonstrated to be able to restore the matching and radiation patterns of both the two antennas as if they were not sensing the presence of the other. Multilayer elliptical cloaks have been also recently proposed in [103] for an interesting combination of cloaking and bandwidth extension functionality. In particular, a twolayer elliptical metasurface made of dog-bone unit-cells has been exploited to decouple both microstrip and freestanding strip monopoles. At the same time, starting from the concepts developed in [104] for the use of coating metasurfaces for frequency bandwidth enhancement, it was demonstrated that the original bandwidth of the two antennas was increased compared to the original case.

Finally, it is worth mentioning that mantle-cloaking solutions for blockage and mutual-coupling reduction have been recently moved to array scenarios. For instance, in [105], a novel compact and high-directivity dual-band radiator with opposite main lobe directions at its two resonance frequencies has been proposed. A first smaller Yagi-Uda antenna, operating at high-frequency, was placed between the driven element of a second larger Yagi-Uda, operating at lower frequencies, intentionally oriented to radiate in the opposite direction compared to 


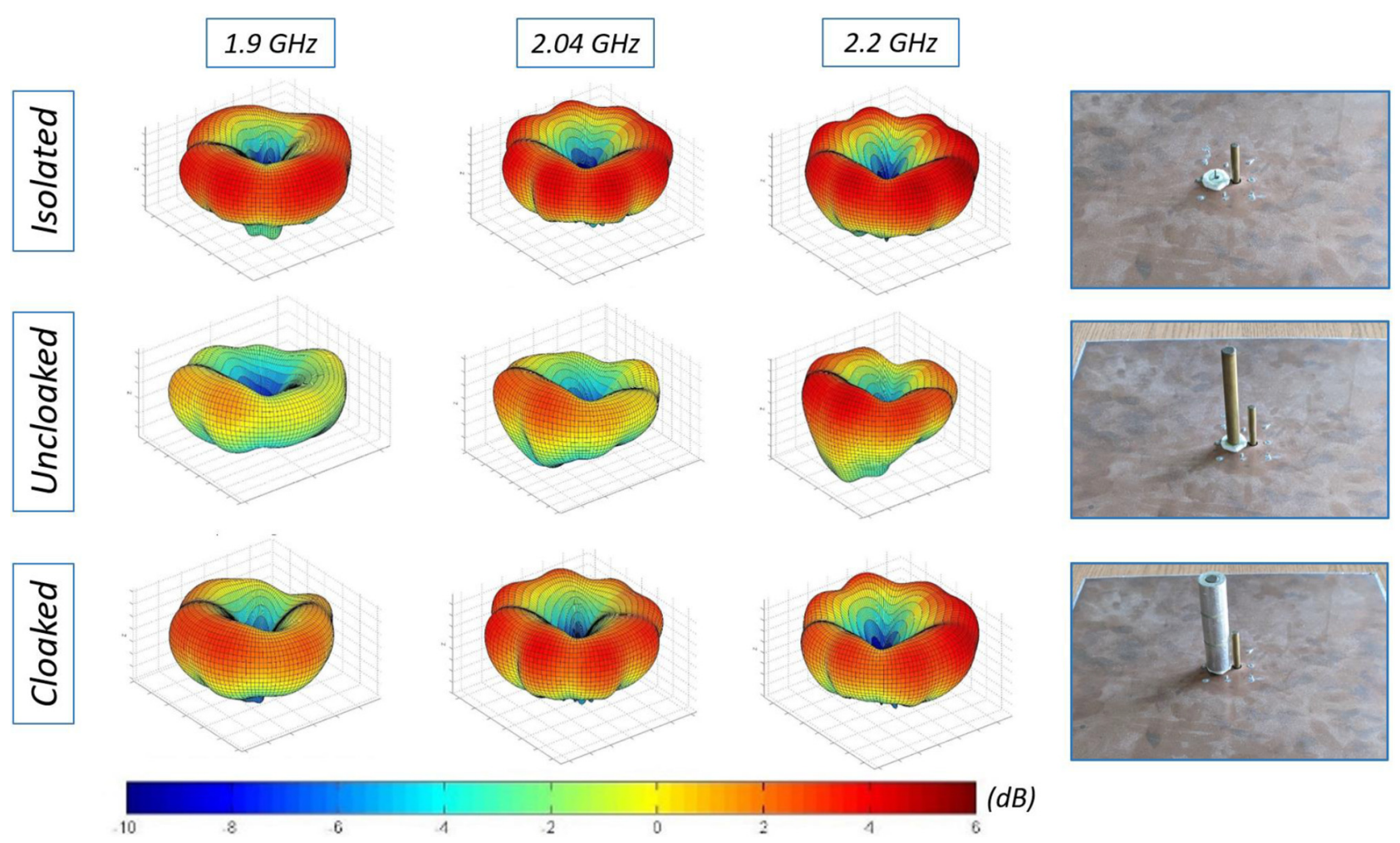

Fig. 13. Measured far-field realized gain patterns of the UMTS monopole at the lowest $(1.9 \mathrm{GHz})$, central $(2.04 \mathrm{GHz})$ and highest $(2.2 \mathrm{GHz})$ frequency of operation of the antenna in the isolated, uncloaked, and cloaked cases. In the insets on the right, a picture of the each considered scenario is shown (isolated, uncloaked, and cloaked) (adapted from [81]).

the previous one. To massively reduce the perturbing effects induced to the first antenna by the second antenna, mantle cloaking devices for the director, reflector, and the driven element of the larger Yagi-Uda were designed. We remark here that this innovative antenna system represents a non-trivial extension of cloaked dipoles since the design of the cloak was complicated by the presence of complex scattering effects from the Yagi-Uda parasitic elements that should be properly taken into account.

More recently, a different array application has been proposed in [106]. Here, the concept of mutual-blockage reduction was applied to design two interleaved antenna array of strip monopoles. Exploiting the elliptical mantle cloaks designed in [101], it was shown that covering each element of the arrays with conformal elliptical mantle cloaks working at the operating frequency of the other array, allow to conceive a compact dual-band array system able to selectively and efficiently scanning the environment in opposite direction.

\section{Advanced cloaking metasurfaces for intelligent antennas}

As extensively discussed in the previous sections, metasurface cloaks have enabled a series of unprecedented applications in the antenna field, enriching antenna performance with several breakthrough functionalities. However, these interesting applications have been obtained exploiting just passive metasurfaces made by patterned metallic ultrathin sheets, usually wound around a dielectric support covering the antenna. In the literature, several different studies proposing the use of electronically loaded metasurfaces to overcome fundamental limitations of passive cloaks have been proposed. For instance, in [107], non-foster devices have been used to broader the cloaking bandwidth. In [108], parity-time cloak with balanced loss and gain have been discussed to cloak electrically large cylinders. Unidirectional mantle cloaks have been proposed to relax some cloaking constraints and, thus, achieve better performances [109]. Finally, external sources driven by independent signal generators have been used to realize an active ultrathin cloak [110].

In the framework of cloaking for antennas, actually, further degrees of freedom can be introduced by loading the cloaking metasurfaces with proper linear and non-linear electronic lumped elements. In this section, we review our recent results in this frame, showing how cloaking metasurfaces loaded by electronic devices allow going beyond the results achieved with passive, linear and timeinvariant mantle cloaks, resulting in a dramatic enhancement of the antenna functionalities. Thanks to the conformal design and extreme feasibility of cloaking metasurfaces, electronic elements can be easily placed onto the surface, making these advanced cloaking metasurfaces easily manufacturable. Specifically, here, two main categories of advanced cloaking metasurfaces are presented: (i) non-linear cloaking metasurfaces for 


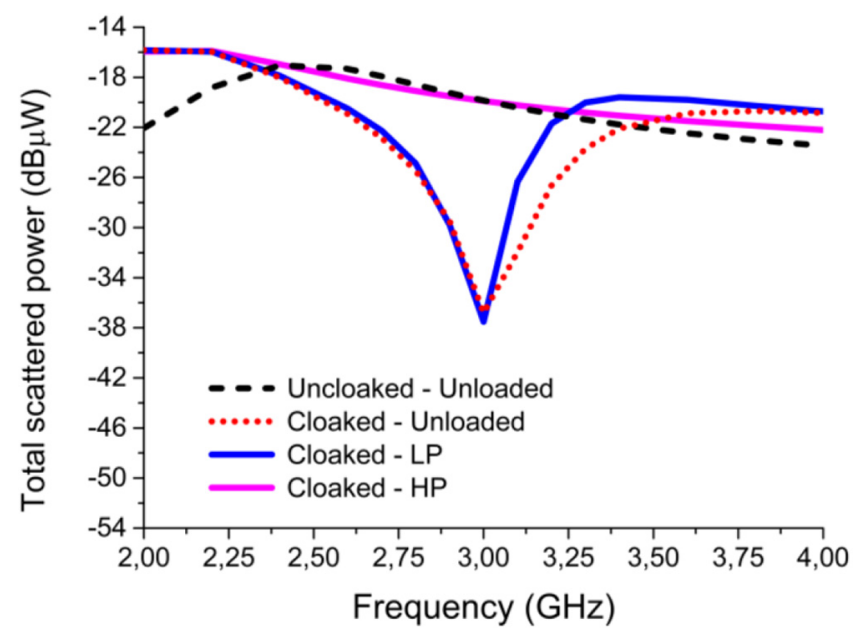

Fig. 14. Performances of a traditional and of a non-linear mantle cloak coating a metallic cylinder in terms of the total scattered power reduction (adapted from [112]).

power-dependent antennas and, (ii) frequency- and timedomain selective mantle cloaks for antenna systems able distinguish between the frequency and waveform characteristics of transmitted/received signals.

\subsection{Non-linear mantle cloaks for antennas}

Following the recent trend in the metamaterial community of exploiting electronic and circuit components to enrich the metasurface functionalities [111], in [112], non-linear cloaking metasurfaces for the design of mantle cloaking devices whose cloaking effect can be turned $\mathrm{ON}$ and $\mathrm{OFF}$ depending on the power level of the impinging electromagnetic field have been proposed. As a first example, the gap between consecutive strips synthesizing a capacitive metasurface able to cloak a coated monopole antenna at $f_{0}=3 \mathrm{GHz}$ has been loaded with a diode pair. As shown in Figure 14, when the antenna is illuminated by a low-power (LP) plane wave, a strong suppression of the antenna scattered power is achieved. Conversely, for a high-power (HP) electromagnetic field, the antenna scattered power response match the scenario where the cloaking coat is not present.

This is due to the intrinsic non-linear power-dependent response of the diodes, which are characterized by a very high equivalent impedance when interacting with LP signals, while exhibit an equivalent low impedance for HP signals. More specifically, the diode pair can be represented by an equivalent circuit, composed of a parallel combination of a high resistance with a low capacitance for LP signals, whereas for HP signals the diode is described by a small forward resistance. Due to the shunt connection between the metasurface gaps, the diode equivalent impedance appears in parallel to the metasurface cloaking surface impedance [112]. Therefore, when illuminated by an HP signal, the loaded cloaking metasurface exhibits a surface impedance close to zero, due to the almost shortcircuiting of the metasurface horizontal strips. Conversely, for LP signals, the loaded metasurface exhibits still a
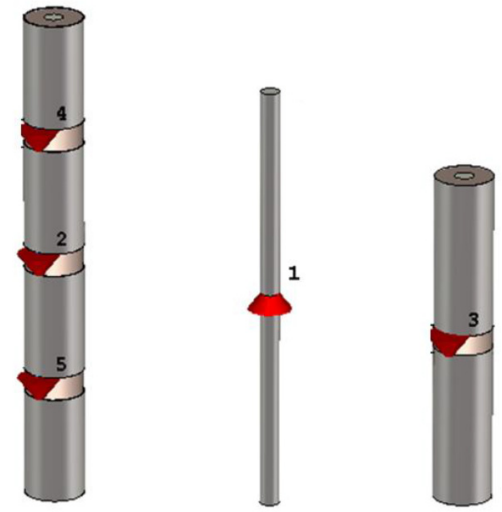

Fig. 15. Sketch of the power-dependent Yagi-Uda antenna coated by non-linear mantle cloaks (adapted from [112]).

capacitive surface impedance, although a slight deviation compared to the cloaking value appears due to the small capacitive effect of the diode (Fig. 14, blue vs red continuous curves). Thus, the metasurface behaves as a perfect electric conductor wrapped around the monopole antenna for HP levels, since the diodes are in the ON state and short circuit the metasurface strips. The cloaking effect is turned OFF in this case. Whereas, for LP level, the diodes are in the OFF state and the metasurface behaves as a cloak, turning ON the cloaking behavior. This operation principle has been used to develop several different applications in the antenna field.

For instance, in [112] the design of a power-dependent Yagi-Uda antenna has been numerically investigated. As well known, the directional pattern characterizing a YagiUda antenna is due to a proper interference between the driven element and the parasitic dipoles, which act as a reflector and director depending on their dimension [94]. To design a power-dependent Yagi-Uda antenna the director and reflector of the antenna have been coated by a nonlinear cloaking coat, as illustrated in Figure 15, able to suppress their scattering contributions at the operating frequency of the antenna. In this way, since the cloaking effect of the parasitic elements of the antenna depends on the power level of the impinging signals, their visibility to the driven element is not always guaranteed. Accordingly to the previous discussion, the passive elements appear hidden for LP signals, whereas are made visible for HP. As shown in Figure 16, in the LP scenario the Yagi-Uda antenna exhibits an omnidirectional pattern due to the turning on of the cloaking effects hiding both reflector and director. For HP signals, instead, the cloaking effect is turned $\mathrm{OFF}$, and the antenna exhibits the standard directive pattern. Interestingly, this innovative configuration finds natural application in radio-base stations where the power levels of the received and transmitted signals are different. In particular, it can be used for the realization of base stations able receiving LP signals from multiple users distributed in the surrounding area, whereas able establishing a directive radio link with other base stations around.

More recently, the possibilities offered by non-linear cloaking metasurfaces have been further investigated enabling unprecedented functionalities in phased antenna 


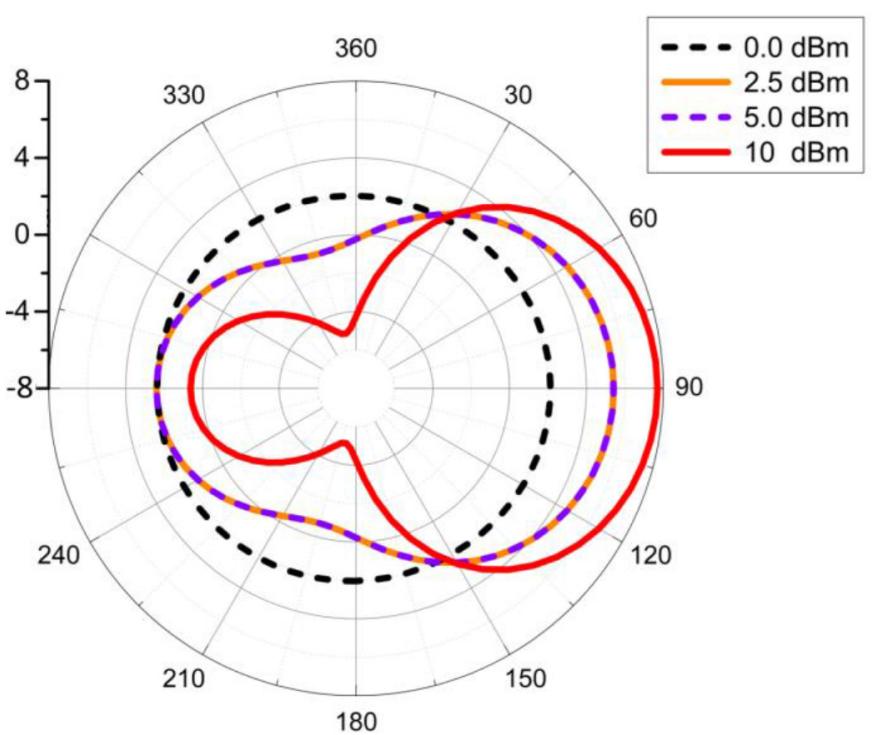

Fig. 16. Polar plot of the power-dependent Yagi-Uda antenna directivity $(\mathrm{dB})$ on the horizontal plane for different values of the antenna input power (adapted from [112]).

arrays [113-115]. In these antenna systems, the scanning capabilities are usually limited by the presence of mutual coupling effects among the elements composing the array [94]. For instance, as illustrated in Figures 17a and 17b for the case of an array of half-wavelength dipole antennas, the embedded element factor of each radiator is usually nonomnidirectional, even though the individual radiating element is omnidirectional if operating in free-space. The mutual coupling effects limit, thus, the scanning range capacity of the array and do not allow the design of a phased array system able to receive from all directions of space. However, this limitation can be circumvented by coating the peripheral elements of the array, except the central one, with a non-linear cloak. In Figures $17 \mathrm{c}$ and $17 \mathrm{~d}$ the behavior of a $3 \times 3$ array of half-wavelength dipole antennas coated by a non-linear cloak for the LP and HP scenarios is reported. Referring to $[114,115]$ for the details of the non-linear cloak design, the mantle cloak applied to the eight peripheral elements can suppress their scattering contributions at their resonant frequency. As can be appreciated (Fig. 17c), in the HP scenario (i.e., in transmitting mode) the array exhibits the usual highlydirective radiation pattern, since the invisibility effect of the cloaks is turned OFF. In the LP scenario of Figure $17 \mathrm{~d}$ (i.e., in receiving mode), instead, the cloaking effect turns $\mathrm{ON}$, and the peripheral elements are made invisible to the central one. The embedded element factor of the central element is thus omnidirectional, and the array is able to receive from all directions in the plane of the array. Therefore, the proposed device could be particularly useful for designing passive radars able to receive low scattered signals from all the directions to identify possible targets and, at the same time, are able to scan selectively the surrounding area.

The discussed radiating systems represent just some preliminary benchmark examples of the possibilities offered by the proposed non-linear cloaking metasurface. Exploiting other metasurface unit-cell configurations overcoming the fundamental limitation of passive and linear metasurfaces [115], further novel radiating systems with enhanced functionalities could be even envisioned.

\subsection{Frequency- and time-domain selective mantle cloaks}

The functionalities of cloaking metasurfaces can be enriched even more by loading them not only with nonlinear discrete components, as in the previous cases, but by introducing more elaborated lumped-element circuit. For instance, the novel concept of a coating metasurface able switching its cloaking functionality ON and OFF depending on both the frequency- and time-domain characteristics of the imping electromagnetic wave has been recently discussed [116,117].

Recently, in $[118,119]$ so-called waveform-selective metasurfaces exhibiting different responses depending on the waveform of the incoming signal have been introduced. Here, the metasurface properties depend not only on the frequency characteristics of the incoming signals, as usually happens due to the inherent dispersion of the structure, but also on the time duration of the impinging wave. This anomalous behavior is achieved thanks to the introduction of a peculiar lumped element circuit loading the metasurface unit-cells, as the one reported in Figure 18c. The circuit is made of a diode bridge rectifier and passive electronic components such as resistors, capacitors, and inductors. When a signal propagates in the circuit, the diode bridge rectifies it mostly to zero frequency, and the equivalent impedance of the circuit varies depending on the transient response of the RLC load. In the RL series case (as the one depicted in Fig. 18c), a strong electromotive force initially appears due to the presence of the inductance, opposing the flow of the current. However, after some time, this opposing effect weakens, and currents can flow towards the output port [119]. Thus, at the beginning, the lumped element circuit behaves as an open circuit whereas, after some time, it almost behaves as a short-circuit. Therefore, for a short-pulsed signal (PW), the circuit acts as an open load, while for a continuous wave signal (CW) it behaves like a low-resistance load. The opposite behaviour can be achieved by using a complementary RC circuit configuration [120].

This unique waveform-selectivity behavior has been exploited for conceiving an innovative cloaking device [117], such as the one depicted in Figure 18. Here, a halfwavelength dipole antenna is coated by a cloaking metasurface loaded by the previously described RL waveform-selective circuit, which is able to hide the antenna to a PW signal generated by a detecting radar (Fig. 18a), while allowing communication with a base station through a continuous waveform (Fig. 18b). Since antennas have a scattering peak at their resonance [94], the mantle cloak has been designed to hide the antenna at its resonant frequency $f_{0}$. In order to make the cloak able distinguishing between the two different signals, an inductive metasurface made of meander unit-cells has been designed. 

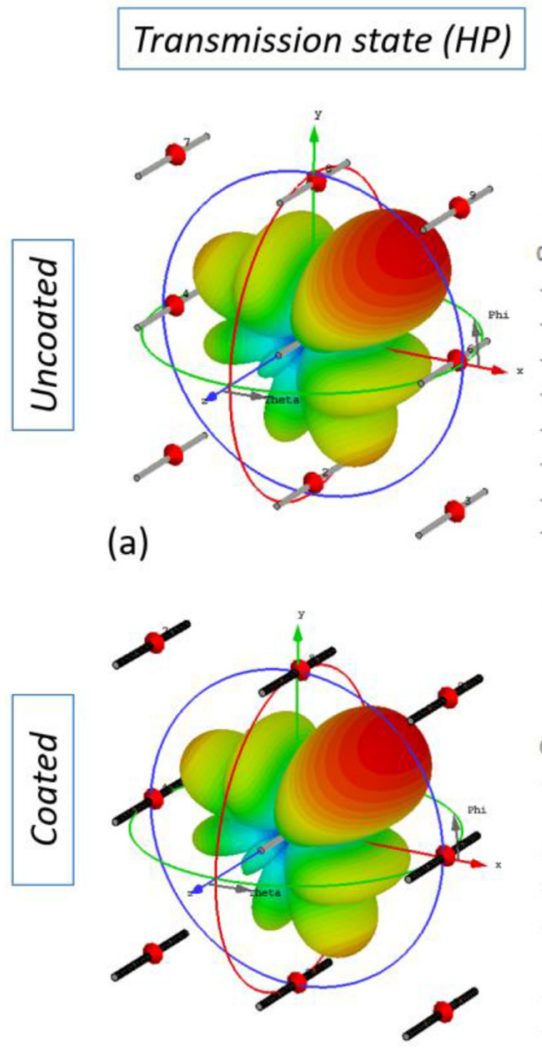

(c)

\section{Reception state (LP)}

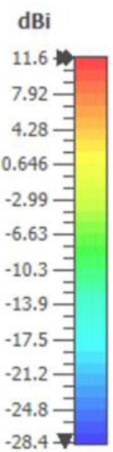

(b)
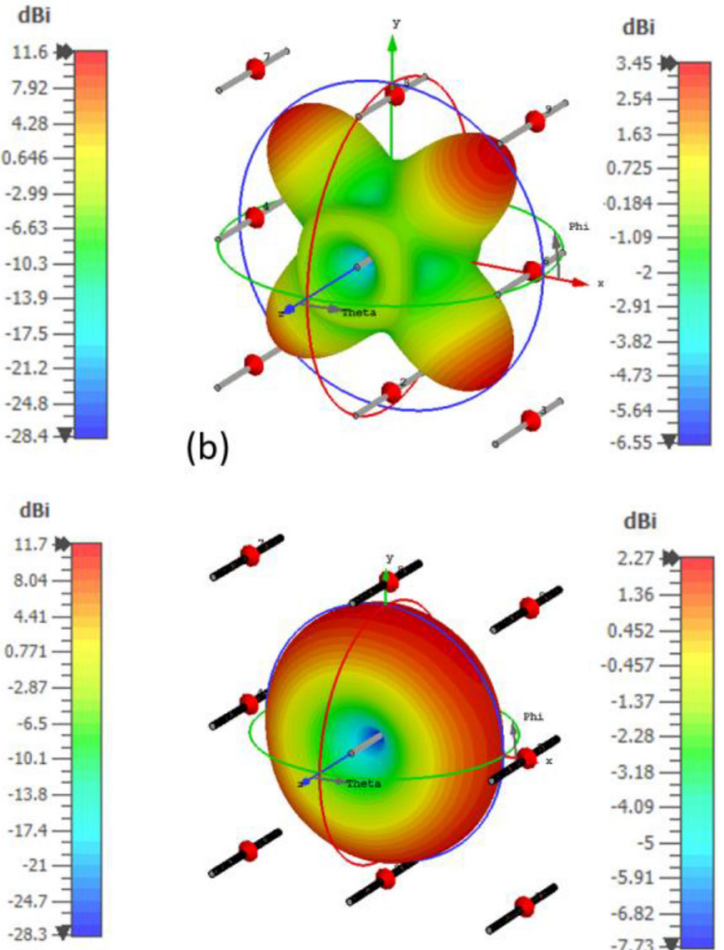

(d)

Fig. 17. Different radiation patterns of a phased array of half-wavelength dipole antennas, when the array elements are excited to obtain the main beam directed in a given direction. (a) and (b) External elements without the cloaking devices (uncoated case): (a) radiation pattern of the array when transmitting HP signals; (b) embedded central element pattern when receiving LP signals. (c) and (d) External elements with the cloaking devices (coated case): (c) radiation pattern of the array when transmitting HP signals;

(d) embedded central element pattern when receiving LP signals (adapted from [115]).

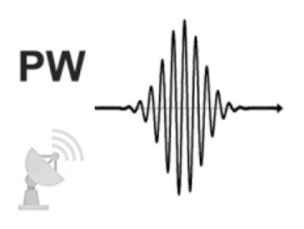

(a)
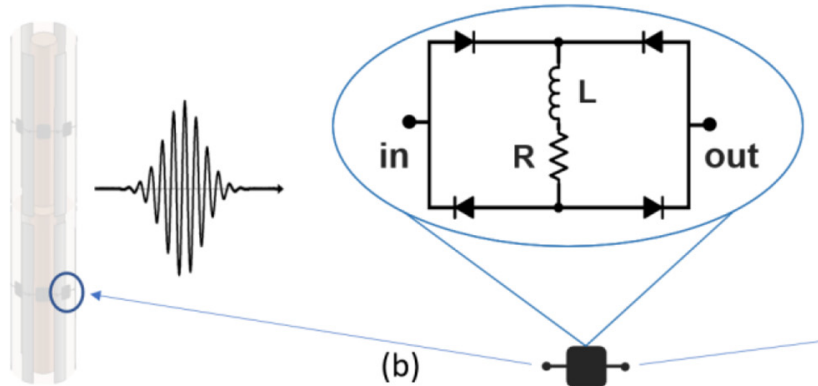

(b)

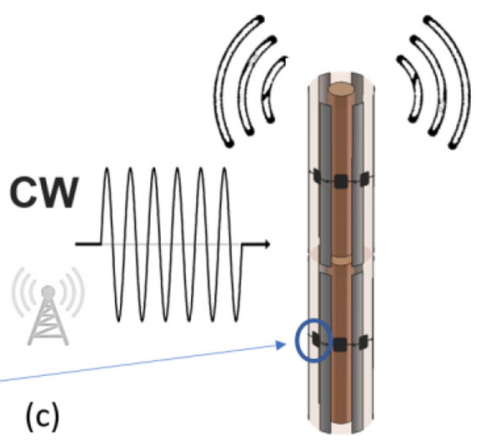

Fig. 18. Sketch of the waveform-selective cloaking concept applied to a wire antenna. (a) Cloaking effect switches ON if illuminated by a pulse signal (PW), and the antenna becomes invisible. (c) Cloaking effects switches OFF if illuminated by a continuous signal $(\mathrm{CW})$, and the antenna operates in the usual way in both reception and transmission. (b) Waveform-selective circuit consisting of a diode bridge rectifier and a series combination of an inductance $(\mathrm{L})$ and a resistor $(\mathrm{R})$ connected in parallel to the input and output ports (adapted from [117]).

Referring to [117] for more details on the design of the metasurface, by properly loading the meander of the unitcell with the RL lumped element circuit the metasurface surface impedance is made dependent on the frequencyand time-domain properties of the impinging signal. As can be appreciated in Figure 19, the equivalent surface impedance of the loaded metasurface at $f_{0}$ switches from the cloaking value to a different one. In particular, for a signal with a short pulse width (i.e., PW) the surface impedance assumes a value able to hide the antenna (i.e, 


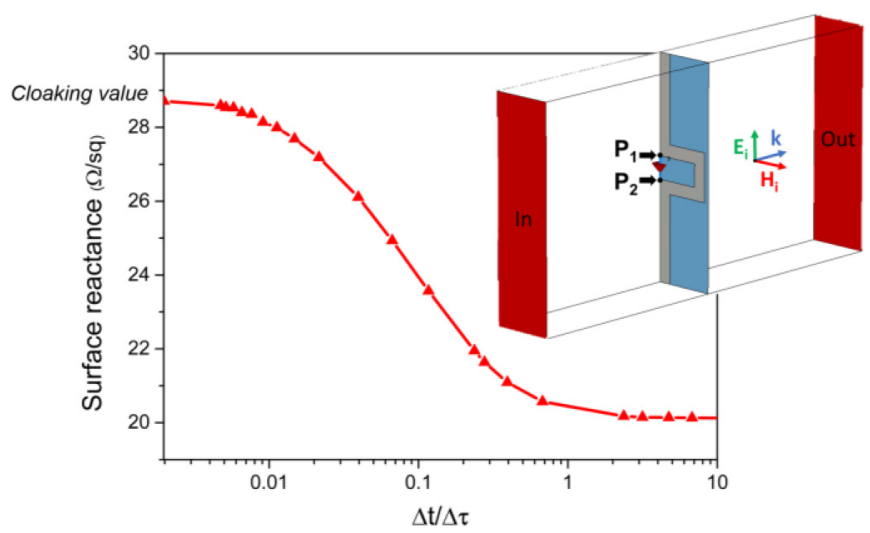

Fig. 19. Response at $f_{0}$ of the cloaking waveform-selective metasurface to a Gaussian pulse modulated by a cosine function at $f_{0}$ varying its pulse width $(\Delta \mathrm{t})$ normalized to the time constant of the loading circuit $(\Delta \tau=\mathrm{L} / \mathrm{R})$ (adapted from [117]).

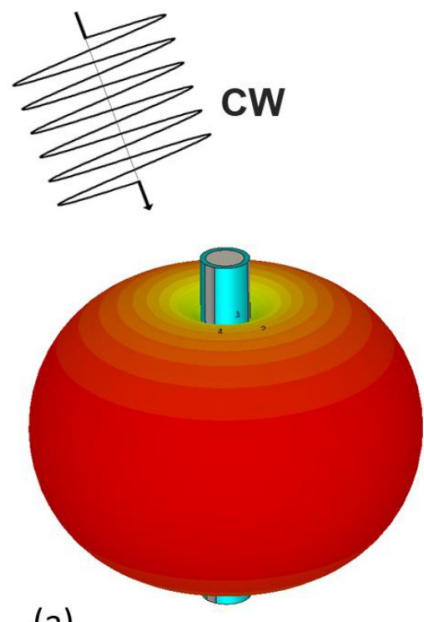

(a)

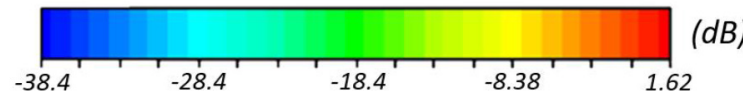

Fig. 20. Realized gain patterns of the antenna coated by the waveform-selective cloak when the dipole is excited by (a) a CW or (b) a PW signal (adapted from [117]).

$Z_{s}=j 29.9 \Omega$ /sq.), while for a long pulse width signal (i.e., $\mathrm{CW}$ ) the surface impedance switches to a value (i.e., $Z_{s}=j 20.1 \Omega$ /sq.) not interfering with the antenna functionality. This switching behavior is due to the open/short-circuiting of the unit-cells meander that, thanks to the presence of the loading circuit and, thus, to the dynamic transformation of its geometrical characteristics, induce a variation on the electromagnetic response of the metasurface. Hence, when analyzing the realized gain patterns of the coated antenna (Fig. 20), we can see that for $\mathrm{CW}$ signals the cloaking effect is turned $\mathrm{OFF}$ and a standard donut-like pattern is achieved (Fig. 20a). For a PW signal, instead, the amplitude of the realized gain is massively reduced due to the cloaking effect coming into place (Fig. 20b).
Overall, the proposed configuration paves the way to novel antenna systems equipped with unconventional functionalities, not only limited to reconfigurable cloaking devices [121], which can expand the ever-growing field of metasurfaces enabling unusual operations.

\section{Conclusions and outlook}

In the last decades, a great effort has been put forward by the research community in translating the fascinating possibilities paved by metamaterial-based cloaking techniques into realistic applications. We have shown that, thanks to their advantages in terms of feasibility and suitability with respect to different techniques, invisibility devices based on the mantle cloaking approach have led to a series of unprecedented applications in antenna and array design. This framework has proved to be one of the most prolific among the realistic scenarios of cloaking proposed in the last years, changing the design paradigm of some antenna systems. In particular, we have reviewed some of the most significant works published in the recent literature showing the great potentialities of mantle cloaking devices for many applications, such as antenna blockage reduction, design of extremely compact terrestrial and satellite communication systems, tuning of the scattering and absorption properties of receiving antennas, cloaking of supporting structure for the restoring the original electric and radiative characteristics of radiating devices. The experimental results demonstrate that the cloaking technology applied to antenna systems is now mature even for radio-frequency industrial applications [122], in view of the next-generation radars and scatteringsignature reduction/control systems of crowded antenna platform.

Moreover, we have shown that other promising and interesting cloaking antenna scenarios are expected to spread in the near future. Some of our recent results on advanced cloaking metasurfaces prove that further exciting possibilities are enabled by loading antenna coating metasurfaces with non-linear electronic components and circuits. Among the recently developed applications, we have discussed the further flexibility introduced by cloaking metasurfaces loaded with PIN diodes in antenna systems design. Furthermore, we have shown that electronic components loading the antenna coating device may introduce even more degrees of freedom by tailoring the antenna response to the waveform. The antenna capabilities may be enriched even more through the use of cloaking devices integrating time-modulated metasurfaces [123], further paving the way to a new generation of intelligent antennas.

We expect that the recent boost in the integration of micro and nanoelectronic components onto metasurfaces will further expand this field, allowing conceiving tunable, reconfigurable, and programmable cloaking devices for radiating systems, towards antennas that are made selfadaptive and cognitive and, thus, able modifying their characteristics such as polarization, frequency of operation, scattering signature, radiation pattern shape, etc. In particular, we can conceive antennas able to sense the 
characteristics of the external electromagnetic environment and modifying their characteristics accordingly to improve or modifying their performances. Compared to current solutions based on signal-processing, in this scenario, the antenna reconfigurability will be due to the intelligence of the coating metasurface, with evident advantages in terms of speed, flexibility of the operation and system integration. The possibility of integrating the intelligence on the coating metasurface physical level will also allow combining the signal process operation with the coating capability giving even more reconfiguration possibilities.

This work has been developed in the frame of the activities of the Project MANTLES, funded by the Italian Ministry of University and Research under the PRIN 2017 Program (protocol number 2017BHFZKH).

\section{References}

1. V.G. Veselago, The electrodynamics of substances with simultaneously negative values of $\varepsilon$ and $\mu$, Sov. Phys. Uspekhi 10, 509 (1968)

2. N. Engheta, R.W. Ziolkowski, Metamaterials: physics and engineering explorations (John Wiley \& Sons \& IEEE Press, Piscataway, New Jersey, 2006)

3. G.V. Eleftheriades, K.G. Balmain, Negative-refraction metamaterials: fundamental principles and applications (John Wiley \& Sons \& IEEE Press, Hoboken, New Jersey, 2005)

4. A.K. Sarychev, V.M. Shalaev, Electrodynamics of metamaterials (World Scientific, Singapore, 2007)

5. T.J. Cui, D.R. Smith, R. Liu, Metamaterials: theory, design, and applications (Springer US, Boston, MA, 2010)

6. F. Capolino, Applications of metamaterials (CRC Press, Boca Raton, FL, 2017)

7. F. Bilotti, C. Vegni, Design of high-performing microstrip receiving gps antennas with multiple feeds, IEEE Antennas Wirel. Propag. Lett. 9, 248 (2010)

8. A.O. Cakmak, K. Aydin, E. Colak, Z. Li, F. Bilotti, L. Vegni, E. Ozbay, Enhanced transmission through a subwavelength aperture using metamaterials, Appl. Phys. Lett. 95, 052103 (2009)

9. F. Bilotti, A. Toscano, L. Vegni, Very fast design formulas for microwave nonhomogeneous media filters, Microw. Opt. Technol. Lett. 22, 218 (1999)

10. B.J. Hoenders, Existence of invisible nonscattering objects and nonradiating sources, J. Opt. Soc. Am. A 14, 262 (1997)

11. A.D. Boardman, K. Marinov, N. Zheludev, V.A. Fedotov, Dispersion properties of nonradiating configurations: Finitedifference time-domain modeling, Phys. Rev. E 72, 036603 (2005)

12. M. Kerker, Invisible bodies, J. Opt. Soc. Am. 65, 376 (1975)

13. H. Chew, M. Kerker, Abnormally low electromagnetic scattering cross sections, J. Opt. Soc. Am. 66, 445 (1976)

14. H. Kurss, Minimum-scattering antennas, IEEE Trans. Antennas Propag. 13, 671 (1965)

15. N.G. Alexopoulos, N.K. Uzunoglu, Electromagnetic scattering from active objects: invisible scatterers, Appl. Opt. 17, 235 (1978)
16. P.S. Kildal, A.A. Kishk, A. Tengs, Reduction of forward scattering from cylindrical objects using hard surfaces, IEEE Trans. Antennas Propag. 44, 1509 (1996)

17. F. Monticone, A. Alù, Invisibility exposed: physical bounds on passive cloaking, Optica 3, 718 (2016)

18. J.B. Pendry, D. Schurig, D.R. Smith, Controlling electromagnetic fields, Science 312, 1780 (2006)

19. U. Leonhardt, Optical conformal mapping, Science 312, 1777 (2006)

20. V.M. Shalaev, Physics: transforming light, Science 322, 384 (2008)

21. U. Leonhardt, To invisibility and beyond, Nature 471, $292(2011)$

22. B. Zhang, Electrodynamics of transformation-based invisibility cloaking, Light Sci. Appl. 1, e32 (2012)

23. E.J. Post, General covariance in electromagnetism (Springer, Berlin, Heidelberg, 1967), pp. 102

24. A.J. Ward, J.B. Pendry, Refraction and geometry in Maxwell's equations, J. Mod. Opt. 43, 773 (1996)

25. F.L. Teixeira, W.C. Chew, Differential forms, metrics, and the reflectionless absorption of electromagnetic waves, J. Electromagn. Waves Appl. 13, 665 (1999)

26. D. Schurig, J.B. Pendry, D.R. Smith, Transformationdesigned optical elements, Opt. Express 15, 14772 (2007)

27. Z. Ruan, M. Yan, C.W. Neff, M. Qiu, Ideal cylindrical cloak: Perfect but sensitive to tiny perturbations, Phys. Rev. Lett. 99, 113903 (2007)

28. J.S. Toll, Causality and the dispersion relation: logical foundations, Phys. Rev. 104, 1760 (1956)

29. U. Leonhardt, T. Tyc, Broadband invisibility by nonEuclidean cloaking, Science 323, 110 (2009)

30. J. Perczel, T. Tyc, U. Leonhardt, Invisibility cloaking without superluminal propagation, New J. Phys. 13, 083007 (2011)

31. P. Alitalo, H. Kettunen, S. Tretyakov, Cloaking a metal object from an electromagnetic pulse: a comparison between various cloaking techniques, J. Appl. Phys. 107, 034905 (2010)

32. D. Schurig, J.J. Mock, B.J. Justice, S.A. Cummer, J.B. Pendry, A.F. Starr, D.R. Smith, Metamaterial electromagnetic cloak at microwave frequencies, Science 314, 977 (2006)

33. J. Li, J.B. Pendry, Hiding under the carpet: a new strategy for cloaking, Phys. Rev. Lett. 101, 203901 (2008)

34. R. Liu, C. Ji, J.J. Mock, J.Y. Chin, T.J. Cui, D.R. Smith, Broadband ground-plane cloak, Science 323, 366 (2009)

35. T. Ergin, N. Stenger, P. Brenner, J.B. Pendry, M. Wegener, Three-dimensional invisibility cloak at optical wavelengths, Science 328, 337 (2010)

36. P. Alitalo, O. Luukkonen, L. Jylhä, J. Venermo, S.A. Tretyakov, Transmission-line networks cloaking objects from electromagnetic fields, IEEE Trans. Antennas Propag. 56, 416 (2008)

37. P. Alitalo, C.A. Valagiannopoulos, S.A. Tretyakov, Simple cloak for antenna blockage reduction, in IEEE Antennas and Propagation Society, AP-S International Symposium (Digest) (2011), pp. 669-672

38. P. Alitalo, S. Ranvier, J. Vehmas, S. Tretyakov, A microwave transmission-line network guiding electromagnetic fields through a dense array of metallic objects, Metamaterials 2, 206 (2008) 
39. P. Alitalo, S. Tretyakov, Electromagnetic cloaking with metamaterials, Mater. Today 12, 22 (2009)

40. P. Alitalo, F. Bongard, J.F. Zürcher, J. Mosig, S. Tretyakov, Experimental verification of broadband cloaking using a volumetric cloak composed of periodically stacked cylindrical transmission-line networks, Appl. Phys. Lett. 94, 014103 (2009)

41. S. Tretyakov, P. Alitalo, O. Luukkonen, C. Simovski, Broadband electromagnetic cloaking of long cylindrical objects, Phys. Rev. Lett. 103, 103905 (2009)

42. J. Vehmas, P. Alitalo, S.A. Tretyakov, Experimental demonstration of antenna blockage reduction with a transmission-line cloak, IET Microw. Antennas Propag. 6, $830(2012)$

43. J. Vehmas, P. Alitalo, S.A. Tretyakov, Transmission-line cloak as an antenna, IEEE Antennas Wirel. Propag. Lett. 10, 1594 (2011)

44. A. Alù, N. Engheta, Achieving transparency with plasmonic and metamaterial coatings, Phys. Rev. E 72, 016623 (2005)

45. A. Alù, Mantle cloak: invisibility induced by a surface, Phys. Rev. B 80, 245115 (2009)

46. A. Alù, N. Engheta, Cloaking a sensor, Phys. Rev. Lett. 102 , 233901 (2009)

47. A. Alù, N. Engheta, Cloaking a receiving antenna or a sensor with plasmonic metamaterials, Metamaterials 4, 153 (2010)

48. R. Fleury, J. Soric, A. Alù, Physical bounds on absorption and scattering for cloaked sensors, Phys. Rev. B 89, 045122 (2014)

49. C.F. Bohren, D.R. Huffman, Absorption and scattering of light by small particles (Wiley, Weinheim, 1998)

50. A. Alù, N. Engheta, Theory and potentials of multi-layered plasmonic covers for multi-frequency cloaking, New J. Phys. 10, $115036(2008)$

51. J.C. Soric, A. Monti, A. Toscano, F. Bilotti, A. Alù, Multiband and wideband bilayer mantle cloaks, IEEE Trans. Antennas Propag. 63, 3235 (2015)

52. C.A. Valagiannopoulos, P. Alitalo, S.A. Tretyakov, On the minimal scattering response of PEC cylinders in a dielectric cloak, IEEE Antennas Wirel. Propag. Lett. 13, 403 (2014)

53. C.Y. Tay, Z.N. Chen, Azimuthally inhomogeneous metasurface cloak for cylindrical objects, IEEE Trans. Antennas Propag. 69, 254 (2021)

54. A. Alù, N. Engheta, Effects of size and frequency dispersion in plasmonic cloaking, Phys. Rev. E 78, 045602(R) (2008)

55. A. Monti, L. Tenuti, G. Oliveri, A. Alù, A. Massa, A. Toscano, F. Bilotti, Design of multi-layer mantle cloaks, in 2014 8th International Congress on Advanced Electromagnetic Materials in Microwaves and Optics, METAMATERIALS 2014 (Institute of Electrical and Electronics Engineers Inc., 2014), pp. 214-216

56. L. Tenuti, G. Oliveri, A. Monti, F. Bilotti, A. Toscano, A. Massa, Design of mantle cloaks through a System-by-Design approach, in 2016 10th European Conference on Antennas and Propagation, EuCAP 2016 (Institute of Electrical and Electronics Engineers Inc., 2016)

57. H. Younesiraad, M. Bemani, S. Nikmehr, Scattering suppression and cloak for electrically large objects using cylindrical metasurface based on monolayer and multilayer mantle cloak approach, IET Microw. Antennas Propag. 13, 278 (2019)
58. A. Alù, N. Engheta, Plasmonic materials in transparency and cloaking problems: mechanism, robustness, and physical insights, Opt. Express 15, 3318 (2007)

59. A. Alù, N. Engheta, Multifrequency optical invisibility cloak with layered plasmonic shells, Phys. Rev. Lett. 100, 113901 (2008)

60. S. Tricarico, F. Bilotti, A. Alù, L. Vegni, Plasmonic cloaking for irregular objects with anisotropic scattering properties, Phys. Rev. E 81, 026602 (2010)

61. A. Alù, D. Rainwater, A. Kerkhoff, Plasmonic cloaking of cylinders: finite length, oblique illumination and crosspolarization coupling, New J. Phys. 12, 103028 (2010)

62. M.G. Silveirinha, A. Alù, N. Engheta, Parallel-plate metamaterials for cloaking structures, Phys. Rev. E 75, 036603 (2007)

63. B. Edwards, A. Alù, M.G. Silveirinha, N. Engheta, Experimental verification of plasmonic cloaking at microwave frequencies with metamaterials, Phys. Rev. Lett. 103, 153901 (2009)

64. D. Rainwater, A. Kerkhoff, K. Melin, J.C. Soric, G. Moreno, A. Alù, Experimental verification of three-dimensional plasmonic cloaking in free-space, New J. Phys. 14, 13054 (2012)

65. F. Bilotti, S. Tricarico, L. Vegni, Plasmonic metamaterial cloaking at optical frequencies, IEEE Trans. Nanotechnol. 9, $55(2010)$

66. A. Monti, F. Bilotti, A. Toscano, Optical cloaking of cylindrical objects by using covers made of core-shell nanoparticles, Opt. Lett. 36, 4479 (2011)

67. M. Fruhnert, A. Monti, I. Fernandez-Corbaton, A. Alù, A. Toscano, F. Bilotti, C. Rockstuhl, Tunable scattering cancellation cloak with plasmonic ellipsoids in the visible, Phys. Rev. B 93, 245127 (2016)

68. P.Y. Chen, A. Alù, Mantle cloaking using thin patterned metasurfaces, Phys. Rev. B 84, 205110 (2011)

69. Y.R. Padooru, A.B. Yakovlev, P.Y. Chen, A. Alù, Analytical modeling of conformal mantle cloaks for cylindrical objects using sub-wavelength printed and slotted arrays, J. Appl. Phys. 112, 034907 (2012)

70. J.C. Soric, P.Y. Chen, A. Kerkhoff, D. Rainwater, K. Melin, A. Alù, Demonstration of an ultralow profile cloak for scattering suppression of a finite-length rod in free space, New J. Phys. 15, 033037 (2013)

71. Z.H. Jiang, D.H. Werner, Exploiting metasurface anisotropy for achieving near-perfect low-profile cloaks beyond the quasi-static limit, J. Phys. D Appl. Phys. 46, 505306 (2013)

72. A. Monti, J.C. Soric, A. Alù, A. Toscano, F. Bilotti, Anisotropic mantle cloaks for TM and TE scattering reduction, IEEE Trans. Antennas Propag. 63, 1775 (2015)

73. R.S. Schofield, J.C. Soric, D. Rainwater, A. Kerkhoff, A. Alù, Scattering suppression and wideband tunability of a flexible mantle cloak for finite-length conducting rods, New J. Phys. 16, 63063 (2014)

74. S. Vellucci, A. Monti, A. Toscano, F. Bilotti, Scattering manipulation and camouflage of electrically small objects through metasurfaces, Phys. Rev. Appl. 7, 034032 (2017)

75. S. Tretyakov, Analytical modeling in applied electromagnetics (Artech House, Norwood, MA, USA, 2003)

76. S.A. Tretyakov, Metasurfaces for general transformations of electromagnetic fields, Philos. Trans. R Soc. A Math. Phys. Eng. Sci. 373, 20140362 (2015) 
77. G. Labate, A. Alù, L. Matekovits, Surface-admittance equivalence principle for nonradiating and cloaking problems, Phys. Rev. A 95, 063841 (2017)

78. G. Labate, S. Podilchak, L. Matekovits, Analytical synthesis of dispersive mantle cloaks for metallic cylinders: a zero contrast methodology, arXiv:1704.03323 (2017)

79. C. Balanis, Advancead engineering electromagnetics (1989), pp. 329-343

80. S. Vellucci, A. Monti, G. Oliveri, A. Massa, A. Toscano, F. Bilotti, Scattering camouflage and manipulation using metasurfaces, in 2016 10th International Congress on Advanced Electromagnetic Materials in Microwaves and Optics, Metamaterials 2016 (2016)

81. A. Monti, J. Soric, M. Barbuto, D. Ramaccia, S. Vellucci, F. Trotta, A. Alù, A. Toscano, F. Bilotti, Mantle cloaking for co-site radio-frequency antennas, Appl. Phys. Lett. 108, 113502 (2016)

82. O. Luukkonen, C. Simovski, G. Granet, G. Goussetis, D. Lioubtchenko, A.V. Räisänen, S.A. Tretyakov, Simple and accurate analytical model of planar grids and highimpedance surfaces comprising metal strips or patches, IEEE Trans. Antennas Propag. 56, 1624 (2008)

83. H. Hashemi, B. Zhang, J.D. Joannopoulos, S.G. Johnson, Delay-bandwidth and delay-loss limitations for cloaking of large objects, Phys. Rev. Lett. 104, 253903 (2010)

84. J.C. Soric, R. Fleury, A. Monti, A. Toscano, F. Bilotti, A. Alù, Controlling scattering and absorption with metamaterial covers, IEEE Trans. Antennas Propag. 62, 4220 (2014)

85. D.H. Kwon, D.H. Werner, Restoration of antenna parameters in scattering environments using electromagnetic cloaking, Appl. Phys. Lett. 92, 113507 (2008)

86. P.S. Kildal, Artificially soft and hard surfaces in electromagnetics, IEEE Trans. Antennas Propag. 38, 1537 (1990)

87. P.S. Kildal, E. Rajo-Iglesias, Mushroom surface cloaks for making struts invisible, in IEEE Antennas and Propagation Society, AP-S International Symposium (Digest) (2007), pp. $869-872$

88. M. Riel, Y. Brand, Y. Demers, P. De Maagt, Performance improvements of center-fed reflector antennas using low scattering struts, IEEE Trans. Antennas Propag. 60, 1269 (2012)

89. S. Vellucci, A. Monti, M. Barbuto, A. Toscano, F. Bilotti, Satellite applications of electromagnetic cloaking, IEEE Trans. Antennas Propag. 65, 4931 (2017)

90. W.A. Shiroma, L.K. Martin, J.M. Akagi, J.T. Akagi, B.L. Wolfe, B.A. Fewell, A.T. Ohta, CubeSats: a bright future for nanosatellites, Cent. Eur. J. Eng. 1, 9 (2011)

91. D. Selva, D. Krejci, A survey and assessment of the capabilities of Cubesats for Earth observation, Acta Astronaut. 74, 50 (2012)

92. S. Vellucci, A. Monti, M. Barbuto, A. Toscano, F. Bilotti, Use of mantle cloaks to increase reliability of satellite-toground communication link, IEEE J. Multiscale Multiphysics Comput. Tech. 2, 168 (2017)

93. S. Vellucci, A. Monti, M. Barbuto, A. Toscano, F. Bilotti, Enhancing the performances of satellite telecommunication systems exploiting electromagnetic cloaking, in 2017 11th International Congress on Engineered Material Platforms for Novel Wave Phenomena, Metamaterials 2017 (2017)

94. C. Balanis, Antenna theory: analysis and design, 4th ed. (Wiley, Hoboken, New Jersey, 2016)
95. S. Tretyakov, Maximizing absorption and scattering by dipole particles, arXiv:1312.0899 (2013)

96. A. Alù, S. Maslovski, Power relations and a consistent analytical model for receiving wire antennas, IEEE Trans. Antennas Propag. 58, 1436 (2010)

97. A. Monti, J. Soric, A. Alu, F. Bilotti, A. Toscano, L. Vegni, Overcoming mutual blockage between neighboring dipole antennas using a low-profile patterned metasurface, IEEE Antennas Wirel. Propag. Lett. 11, 1414 (2012)

98. Z.H. Jiang, P.E. Sieber, L. Kang, D.H. Werner, Restoring intrinsic properties of electromagnetic radiators using ultralightweight integrated metasurface cloaks, Adv. Funct. Mater. 25, 4708 (2015)

99. Z.H. Jiang, D.H. Werner, Dispersion engineering of metasurfaces for dual-frequency quasi-three-dimensional cloaking of microwave radiators, Opt. Express 24, 9629 (2016)

100. J.C. Soric, A. Monti, A. Toscano, F. Bilotti, A. Alu, Dualpolarized reduction of dipole antenna blockage using mantle cloaks, IEEE Trans. Antennas Propag. 63, 4827 (2015)

101. H.M. Bernety, A.B. Yakovlev, Reduction of mutual coupling between neighboring strip dipole antennas using confocal elliptical metasurface cloaks, IEEE Trans. Antennas Propag. 63, 1554 (2015)

102. H.M. Bernety, A.B. Yakovlev, Decoupling antennas in printed technology using elliptical metasurface cloaks, J. Appl. Phys. 119, 014904 (2016)

103. G. Moreno, A.B. Yakovlev, H.M. Bernety, D.H. Werner, H. Xin, A. Monti, F. Bilotti, A. Alu, Wideband elliptical metasurface cloaks in printed antenna technology, IEEE Trans. Antennas Propag. 66, 3512 (2018)

104. Z.H. Jiang, M.D. Gregory, D.H. Werner, A broadband monopole antenna enabled by an ultrathin anisotropic metamaterial coating, IEEE Antennas Wirel. Propag. Lett. 10, 1543 (2011)

105. A. Monti, J. Soric, A. Alù, A. Toscano, F. Bilotti, Design of cloaked Yagi-Uda antennas, EPJ Appl. Metamat. 3, 10 (2016)

106. H. Mehrpour Bernety, A.B. Yakovlev, H.G. Skinner, S.Y. Suh, A. Alu, Decoupling and cloaking of interleaved phased antenna arrays using elliptical metasurfaces, IEEE Trans. Antennas Propag. 68, 4997 (2020)

107. P.Y. Chen, C. Argyropoulos, A. Alù, Broadening the cloaking bandwidth with non-foster metasurfaces, Phys. Rev. Lett. 111, 233001 (2013)

108. A. Kord, D.L. Sounas, A. Alù, Active microwave cloaking using parity-time-symmetric satellites, Phys. Rev. Appl. 10, 054040 (2018)

109. D.L. Sounas, R. Fleury, A. Alù, Unidirectional cloaking based on metasurfaces with balanced loss and gain, Phys. Rev. Appl. 4, 014005 (2015)

110. M. Selvanayagam, G.V. Eleftheriades, Experimental demonstration of active electromagnetic cloaking, Phys. Rev. X 3, 041011 (2014)

111. Z. Luo, X. Chen, J. Long, R. Quarfoth, D. Sievenpiper, Nonlinear power-dependent impedance surface, IEEE Trans. Antennas Propag. 63, 1736 (2015)

112. A. Monti, M. Barbuto, A. Toscano, F. Bilotti, Nonlinear mantle cloaking devices for power-dependent antenna arrays, IEEE Antennas Wirel. Propag. Lett. 16, 1727 (2017)

113. A. Monti, M. Barbuto, A. Toscano, F. Bilotti, Powerdependent invisibility devices for antenna arrays, in 2019 URSI International Symposium on Electromagnetic Theory, EMTS 2019 (Institute of Electrical and Electronics Engineers Inc., 2019) 
114. S. Vellucci, A. Monti, M. Barbuto, M. Salucci, G. Oliveri, A. Toscano, F. Bilotti, Non-linear mantle cloaks for selfconfigurable power-dependent phased arrays, in 2020 XXXIIIrd General Assembly and Scientific Symposium of the International Union of Radio Science (IEEE, 2020), pp. 1-3

115. S. Vellucci, A. Monti, M. Barbuto, M. Salucci, G. Oliveri, A. Toscano, F. Bilotti, Overcoming Mantle Cloaking Limits in Antenna Applications through Non-Linear Metasurfaces, in 2020 Fourteenth International Congress on Artificial Materials for Novel Wave Phenomena (IEEE, New York, 2020)

116. S. Vellucci, A. Toscano, F. Bilotti, A. Monti, M. Barbuto, Towards waveform-selective cloaking devices exploiting circuit-loaded metasurfaces, in 2018 IEEE Antennas and Propagation Society International Symposium and USNC/ URSI National Radio Science Meeting, APSURSI 2018Proceedings (Institute of Electrical and Electronics Engineers Inc., 2018), pp. 1861-1862

117. S. Vellucci, A. Monti, M. Barbuto, A. Toscano, F. Bilotti, Waveform-selective mantle cloaks for intelligent antennas, IEEE Trans. Antennas Propag. 68, 1717 (2019)
118. H. Wakatsuchi, D. Anzai, J.J. Rushton, F. Gao, S. Kim, D. F. Sievenpiper, Waveform selectivity at the same frequency, Sci. Rep. 5, 9639 (2015)

119. H. Wakatsuchi, J. Long, D.F. Sievenpiper, Waveform selective surfaces, Adv. Funct. Mater. 29, 7196 (2019)

120. M. Barbuto, D. Lione, A. Monti, S. Vellucci, F. Bilotti, A. Toscano, Waveguide components and aperture antennas with frequency- and time-domain selectivity properties, IEEE Trans. Antennas Propag. 68, 7196 (2020)

121. S. Vellucci, M. Barbuto, A. Monti, A. Toscano, F. Bilotti, Waveform-selective devices for antenna applications, in 2020 XXXIIIrd General Assembly and Scientific Symposium of the International Union of Radio Science (IEEE, 2020), pp. 1-4

122. G. Guarnieri, G. Mauriello, S. Scafe, M. Barbuto, A. Monti, D. Ramaccia, S. Vellucci, A. Tobia, A. Toscano, F. Bilotti, Metamaterials meeting industrial products: a successful example in Italy, in 2016 IEEE Antennas and Propagation Society International Symposium, APSURSI 2016-Proceedings (2016)

123. D. Ramaccia, D.L. Sounas, A. Alù, A. Toscano, F. Bilotti, Doppler cloak restores invisibility to objects in relativistic motion, Phys. Rev. B 95, 075113 (2017)

Cite this article as: Stefano Vellucci, Alessio Monti, Mirko Barbuto, Alessandro Toscano, Filiberto Bilotti, Progress and perspective on advanced cloaking metasurfaces: from invisibility to intelligent antennas, EPJ Appl. Metamat. 8, 7 (2021) 